\title{
1 Glycine acylation and trafficking of a new class of bacterial lipoprotein by a composite secretion
}

2 system.

3 Christopher Icke ${ }^{1}$, Freya J. Hodges ${ }^{1}$, Karthik Puella ${ }^{1}$, Samantha A. McKeand ${ }^{2}$, Jack A. Bryant ${ }^{2}$, Adam

4 F. Cunningham ${ }^{2,3}$, Jeff A. Cole ${ }^{2}$, Ian R. Henderson ${ }^{1 *}$.

5 Institute for Molecular Bioscience, University of Queensland, Brisbane, Australia; ${ }^{2}$ Institute of

6 Microbiology and Infection and ${ }^{3}$ Institute of Immunology and Immunotherapy, University of

7 Birmingham, Birmingham, UK

$8 \quad$ For correspondence: Ian Henderson (i.henderson@uq.edu.au)

9 


\section{Abstract}

11 Protein acylation is critical for many cellular functions including signal transduction, cell division and

12 development. In bacteria, such lipoproteins have important roles in virulence and are therefore potential 13 targets for the development of novel antimicrobials and vaccines. To date, all known bacterial 14 lipoproteins are secreted from the cytosol via the Sec pathway, acylated on an N-terminal cysteine residue through the action of Lgt, Lsp and Lnt, and then targeted to the appropriate cellular location. In the case of Gram-negative bacteria, the lipoprotein trafficking Lol pathway transports the lipoproteins to the outer membrane where most substrate molecules are retained within the cell. Here we identify a new secretion pathway that displays the substrate lipoprotein on the cell surface. We demonstrate that the previously identified E. coli Aat secretion system is a composite system that shares similarity with type I secretion systems and elements of the Lol pathway. Remarkably, during secretion by the Aat system, the AatD subunit acylates the substrate CexE on a highly conserved N-terminal glycine residue (rather than the canonical cysteine). Mutations in AatD or CexE that disrupt glycine acylation interfere with membrane incorporation and trafficking. Our data suggest that CexE is the first member of a new class of glycine-acylated bacterial lipoprotein, while Aat represents a new secretion system that we propose be defined as a lipoprotein secretion system (LSS). 


\section{Introduction}

Protein acylation by the covalent attachment of fatty acids occurs for hundreds of proteins in eukaryotic and prokaryotic organisms. This event confers distinct biochemical properties, enabling acylation to regulate intracellular trafficking, subcellular localization, protein-protein and protein-lipid interactions and are of obvious importance to cell biology. As a consequence, lipoproteins are key components of bacterial pathogens and have been targeted for antibiotic and vaccine development (Dev et al., 1985). In Gram-negative bacteria, lipoproteins are transported across the inner membrane from the cytosol to the periplasm by the Sec pathway. However, in Gram-positive cells lipoproteins can also be secreted by the TAT pathway (Thompson et al., 2010; Widdick et al., 2011). The signal peptide inserts into the inner membrane and a diacylglycerol is attached by Lgt to the sulphur moiety of the invariant cysteine at the +1 position of the lipoprotein. The signal sequence is then cleaved by Lsp exposing the N-terminal amine group of the cysteine for monoacylation by Lnt (Gupta et al., 1993; Masao Tokunaga et al., 1984). Lnt mediated acylation occurs at the free amine moiety of the N-terminal cysteine after signal sequence cleavage by Lsp (Signal Peptidase II). The mature triacylated lipoprotein remains embedded in the inner membrane or is localised to the inner leaflet of the outer membrane by the essential Lol pathway. LolCE, in combination with the ATPase LolD, extracts the triacylated lipoprotein from the inner membrane for transport across the periplasm by LolA, where it is then incorporated into the inner leaflet of the outer membrane by LolB. While the majority remain periplasmically located, in recent years there have been a number of descriptions of surface localised outer membrane lipoproteins (Baldi et al., 2012; Cowles et al., 2012; Konovalova et al., 2014). However, the mechanism of translocation across the outer membrane to the cell surface remains poorly understood.

Previously, we described two outer membrane proteins, CexE and Aap, associated with enterotoxigenic (ETEC) and enteroaggregative Escherichia coli (EAEC), respectively (Crossman et al., 2010; Sheikh et al., 2002). CexE has been implicated in prolonged bacterial shedding and increased severity of infection (Rivas et al., 2020) whereas Aap influences biofilm formation and gut colonisation (Belmont-Monroy et al., 2020; Sheikh et al., 2002). Despite Aap and CexE sharing only $18 \%$ amino 
acid identity, both proteins are secreted by the Aat system (Belmont-Monroy et al., 2020; Nishi et al., 2003; Rivas et al., 2020). The Aat system requires five proteins (AatPABCD) to facilitate protein secretion, two of which bear resemblance to components of the Type 1 secretion system (T1SS); an outer membrane protein (OMP), and a periplasmic adaptor protein (PAP). In contrast to the T1SS, the CexE and Aap substrate molecules are translocated using a two-step mechanism. First Aap/CexE is translocated across the inner membrane into the periplasm by the Sec pathway (Pilonieta et al., 2007). It then enters the Aat system to be secreted across the outer membrane (Belmont-Monroy et al., 2020; Nishi et al., 2003; Rivas et al., 2020).

While further characterising the two-step secretion mechanism of the Aat system, we noticed that during secretion CexE is post-translationally modified by the Aat system and this modification is required for the secretion. Here we reveal that following cleavage of the Sec-dependent signal sequence, the N-terminus of CexE is modified by the addition of an acyl chain. We demonstrate that AatD is a homolog of the apolipoprotein $\mathrm{N}$-acyltransferase (Lnt). We reveal AatD is both necessary and sufficient for monoacylation of CexE and Aap. However, in contrast to Lol lipoprotein substrates, CexE lacks an $\mathrm{N}$-terminal cysteine and instead an invariant glycine is the site of acylation. Furthermore, we demonstrate that the addition of an $\mathrm{N}$-terminal glycine to the coding sequence of a heterologous protein was sufficient for this novel AatD-catalysed acylation event. We propose that Aap and CexE are members of a novel class of lipoprotein that are secreted through the Aat system, which is a conglomeration of the Lol pathway and a T1SS. Thus, we describe a new mechanism of Npalmitoylation. We reveal glycine as a new target of N-palmitoylation and AatD is a new acyltransferase. Consequently, we reveal a new function for acylation - protein secretion.

\section{Results}

\section{Distribution of the Aat system}

The Aat system was first identified in EAEC, where it corresponds to the molecular probe (CVD432) used to define this E. coli pathovar (Baudry et al., 1990; Nishi et al., 2003). In order to determine whether the Aat system was more widespread each of the Aat proteins from ETEC H0407 was used to search the non-redundant protein sequence database using repetitive iterations of the PSI- 
BLAST algorithm. The full Aat system and an Aap or CexE homolog was identified in 826 separate nucleotide accessions (Supplementary Table S1). This revealed that the Aat system is distributed more widely than initially anticipated and is encoded in pathogens with diverse mechanisms of virulence such as ETEC, EAEC, enteropathogenic E. coli, Shiga-toxin producing E. coli, Shigella sp., Salmonella enterica, Citrobacter rodentium, Providencia alcalifaciens and Yersinia entercolitica (Crossman et al., 2010; Petty et al., 2010; Rivas et al., 2020).

To understand the conservation of the Aat system, the organisation of the aat operon was examined for each of the 826 genomes identified above. The relative genomic distance between each of the aat genes and aap/cexE was calculated from their boundaries on the nucleotide accession. From this analysis six separate organisations of the aat operon were identified (Fig 1). In the most common organisation aap/cexE are separated from the aatPABCD operon by at least $1 \mathrm{~kb}$. This accounted for just over half of the aat systems identified. In just under a quarter of aat operons identified, aap/cexE are within $1 \mathrm{~kb}$ of the aatPABCD operon either within 400 bp upstream (18.4\%) or $1 \mathrm{~kb}$ downstream (4.8\%). In $25 \%$ of the aat systems identified aatD was separated from aatPABC by $>1 \mathrm{~kb}$. The most common of these organisations was aatD separated from the other members of the aat system and substrate protein (11.9\%), closely followed by aatD and aap/cexE together but greater than $1 \mathrm{~kb}$ distance from for other aat genes (9.0\%). Finally, in the least common example aatD, aap/cexE and aatPABC were all separated by greater than $1 \mathrm{~kb}(4.1 \%)$. We did not identify any examples of aatD encoded between $39 \mathrm{bp}$ and $1 \mathrm{~kb}$ from the stop codon of aatC. However, despite these differences, in every example the aatPABC genes formed a single operonic unit located with $a a t D$ and $a a p / c e x E$ on a chromosomal pathogenicity island or a large virulence plasmid. These data suggest that AatPABCD are sufficient and necessary to mediate Aap/CexE secretion.

\section{Aat-dependent secretion of CexE}

To test whether all of the aat genes are required for CexE secretion, we constructed single gene deletion mutants of cexE and each aat gene in ETEC H10407 pCfaD. The pCfaD plasmid encodes the $\mathrm{CfaD}$ transcriptional activator under the control of an arabinose inducible promoter allowing constitutive expression of the CfaD-dependent cexE and the aat genes in the presence of arabinose 
107 (Hodson et al., 2017; Pilonieta et al., 2007). SDS-PAGE analysis of culture supernatant fractions 108 collected from ETEC H10407 pCfaD revealed a protein with an apparent molecular mass of $11.8 \mathrm{kDa}$ 109 that could be detected by western blotting with CexE-specific polyclonal antibodies (Fig 2A). In 110 contradistinction to the parent strain, no protein was detected in the culture supernatant fractions derived 111 from cultures of the ETEC H10407 pCfaD aat or cexE mutants (Fig 2A). However, to ensure that the 112 lack of CexE in the culture supernatant was not due to the lack of CexE production, but rather a result of an inability to secrete CexE, the presence of CexE in whole cell lysates was determined. CexE was detected by western blotting with CexE-specific antibodies in whole cell lysates of ETEC H10407 pCfaD and all aat mutants. In contrast, CexE could not be detected in the cexE null mutant (Fig. 2A). From these data we conclude that the Aat system is essential for CexE secretion.

\section{Post-translational modification of Aat substrate molecules}

Unexpectedly, when the whole cell lysates of ETEC H10407 pCfaD and its isogenic aat mutants were examined by western blotting, CexE appeared as two bands in the parental strain and each of the aat mutants except for aatD (Fig. 2B). The upper band had an apparent molecular mass of 12.7 $\mathrm{kDa}(\mathrm{uCexE})$ while the lower band (mCexE) had an apparent molecular mass of $11.8 \mathrm{kDa}$, which is consistent with the secreted form of CexE recovered from the supernatant fractions. As CexE is exported into the periplasm by the Sec pathway (Pilonieta et al., 2007), the inefficient cleavage of a signal peptide could be responsible for the difference in the molecular mass of uCexE compared to mCexE . To investigate this, ETEC H10407 pCfaD was grown in the presence of sodium azide. Sodium azide inhibits SecA to prevent translocation of proteins across the inner membrane and subsequent cleavage of the signal peptide (Huie \& Silhavy, 1995; Oliver et al., 1990). In the absence of sodium azide the mCexE and uCexE forms could be detected in whole cell lysates as observed previously (Fig 2B and C). However, in the presence of sodium azide a protein (proCexE) with an apparent molecular mass of $13.2 \mathrm{kDa}$ was detected (Fig. 2C). These observations suggest that the difference in molecular mass between uCexE and mCexE was not due to inefficient cleavage of the signal peptide but was due to a post translational event mediated by AatD. 

investigations of the Aat system. To determine whether the migration of Aap was altered by AatD, the effect of an aatD deletion on the apparent size of Aap was investigated in EAEC 042. EAEC 042, an aap mutant, an aatD mutant and an aatD mutant complemented with pJNW were grown in DMEMHG; the pJNW plasmid encodes the complete aat operon from EAEC 042. Whole cell lysates of each strain were analysed by SDS-PAGE. As noted above for CexE, the deletion of aatD in EAEC 042 resulted in an apparent increase in size of Aap (Fig 2D). The size change of Aap in the aatD mutant could be restored to the wild-type size by complementation with the pJNW plasmid. From these data we conclude that AatD can modify Aap and CexE post-translationally and that this modification is required for secretion of the mature substrate molecule to the extracellular milieu.

\section{Predicted functions of Aat components}

To assist our understanding of the contribution of each Aat component to the secretion of CexE we constructed hidden Markov-models (HMM) for individual Aat proteins. These were used to search the Uniprot database for distant homologs. In agreement with previous publications, Aat A is a homolog of the trimeric outer membrane protein TolC (Supplementary Fig S1) and AatB is a periplasmic adaptor protein (Supplementary Fig S2), components associated with T1SS and drug efflux pumps (Nishi et al., 2003). In contrast, AatD was not homologous to any T1SS or efflux-associated proteins. Instead, AatD was homologous to apolipoprotein $\mathrm{N}$-acyltransferase (Lnt), which is a member of the carbon-nitrogen hydrolase (C-N hydrolase) family (PF00795) required for bacterial lipoprotein acylation and transport via the Lol system (Fig 3A). Comparison of the C-N hydrolase domain of AatD with other members of PF00795 revealed that AatD forms a clade with Lnt suggesting that they are functionally related. The ATPase domain of AatC is part of a clade containing the ATPase domains of LolD, the ATPase subunit of the LolCDE lipoprotein transporter (Fig 3B). Similarly, AatP is more closely related to LolC and LolE than typical ABC transporters of the T1SS, such as HlyB (Fig 3C). The homology of AatP, AatC, and the Lol lipoprotein trafficking system. 

acylated form of CexE. To test this hypothesis, we used 17-ODYA, an 18-carbon alkyne fatty acid that can be conjugated to an azide linked fluorescent molecule by a copper(I)-catalyzed azide-alkyne cycloaddition (CuAAC) reaction to investigate CexE lipidation. The cexE gene was introduced into a cexE mutant or a cexE aatD double mutant on a plasmid termed pACYC-cexE-6His, which encodes cexE under the transcriptional control of its native promoter. This allowed production of a $\mathrm{C}$-terminally His-tagged variant of CexE. Both strains harbouring pACYC-cexE-6His were grown in the presence or absence of 17-ODYA. The bacterial lipoprotein YraP, was included as a positive control. CexE and YraP were isolated from each culture by virtue of their C-terminal 6xHis-tag by using nickel affinity chromatography. The purified proteins were then linked to an azide derivative of Alexa Fluor 488 using a CuAAC reaction. After the CuAAC reaction the CexE and YraP samples were separated by SDSPAGE and fluorescence was measured (Fig 4). In the presence of AatD and 17-ODYA CexE was fluorescently labelled; in the absence of either 17-ODYA or AatD no fluorescence was observed. Moreover, only mCexE was labelled indicating that $\mathrm{uCexE}$ is an unacylated form of CexE; counter intuitively, the acylated form (mCexE) migrates faster on SDS-PAGE than the unacylated uCexE. As acylated proteins associate with membranes, we examined the cellular compartmentalisation of mCexE (Supplementary Fig S3). ETEC H10407 pCfaD and isogenic aat mutants were grown in LB supplemented with L-arabinose. Subsequently, cells were collected by centrifugation, lysed and the total membrane fraction was harvested by centrifugation. After separation by SDS-PAGE, only mCexE was detectable by western blotting in the membrane fraction of the parent strain and the aatPABC mutants; no mCexE could be detected in the membranes recovered from the aatD mutant (Supplementary Fig S3). In contrast, both mCexE and uCexE could be detected in whole cell lysates of ETEC H10407 pCfaD and only uCexE could be detected in whole cell lysates of the aatD mutant. These data suggest that AatD is an acyltransferase that mediates post-translational modification of CexE by the addition of one or more acyl chains.

To determine whether AatD was sufficient to mediate the acylation of mCexE, plasmids encoding AatD and CexE were introduced into the laboratory strain E. coli BL21(DE3), which does not encode any of the Aat proteins or Aap/CexE. E. coli BL21 was transformed with pET26b-cexE and 
pACYC-aatD or their respective empty vector controls pET26b and pACYCDuet-1. These strains were grown in LB and the production of AatD and CexE was induced by IPTG. The production of CexE was monitored via western blotting (Fig 5). CexE was not detected in strains that contained pET26b. uCexE was produced in the absence of AatD at the size expected for the unmodified form after cleavage of the signal sequence. However, when CexE and AatD were produced together, mCexE migrated further (Fig

5). These data suggest AatD was solely responsible for the modification of CexE.

\section{Catalytic residues of AatD}

The C-N hydrolase family of proteins have a known conserved catalytic triad, which in the case of Lnt is E267, K335, and C387. Another Lnt residue E343 helps to stabilise the catalytic site (VidalIngigliardi et al., 2007). Mutation of any of these residues to alanine results in a loss of Lnt function (Gélis-Jeanvoine et al., 2015). To assess the conservation of the catalytic residues the AatD sequence of EAEC 042 and ETEC H10407 and Lnt from E. coli MG1655 were aligned using Clustal Omega. Each of the residues associated with the catalytic site were conserved in both versions of AatD (Fig 6A). The C-N hydrolase catalytic triad is conserved in AatD: E217; K278; and C325. Also, Lnt E343 is conserved at position E286 in AatD. Further AatD sequences were identified using PSI-BLAST. These sequences were aligned and a WebLogo was produced for the regions flanking each of the catalytic residues; the four residues were $100 \%$ conserved in all AatD sequences identified. In addition, when a structure of AatD predicted by Phyre2 (Kelley et al., 2016) was compared to Lnt (PDB: 5N6L) (Wiktor et al., 2017) the four AatD residues (E217, K278, E286, and C325) were superimposed on the Lnt catalytic triad suggesting that these residues were required for acylation of CexE (Fig 6B and 6C). To test this hypothesis, pACYC-aatD derivatives encoding mutant derivatives of AatD with alanine substitutions at the conserved E217, K276, E286, or C325 were introduced into E. coli BL21(DE3) pET26b-cexE. Expression of AatD and CexE were induced by the addition of IPTG during growth in LB. As expected, mCexE was only present in the strain encoding the wild-type aatD sequence whereas only unmodified uCexE was detected in mutants harbouring the E217A, K276A, E286A, or C325A (Fig 6D). These observations suggest that the mechanism of protein acylation by AatD and Lnt are similar. 


\section{An N-terminal glycine is required for acylation}

Due to the homology and functional similarity between AatD and Lnt, a likely site for acylation of CexE is the N-terminal amino acid immediately after the signal sequence. SignalP predictions suggested that CexE possesses a Sec-dependent signal sequence cleaved by Signal Peptidase I between alanine at position 19 and glycine at position 20 (Fig. 7A and Supplementary Table S2). N-terminal amino acid sequencing of uCexE revealed the amino acid sequence GGGNS confirming that glycine at position 20 formed the $\mathrm{N}$-terminal amino acid of the processed protein. However, bioinformatic analyses of the amino acid sequences of 224 distinct CexE homologs failed to identify in their signal sequences the presence of a 'lipobox' (Supplementary Fig S4); lipoboxes are required for recognition of lipoproteins by the Lol system and thus acylation by Lnt (Babu et al., 2006). Further analyses revealed limited sequence identity between the signal sequences of the $224 \mathrm{CexE}$ homologs indicating that an alternative lipobox is not present. Moreover, neither CexE nor any other homolog possessed a cysteine residue adjacent to the signal sequence; for bacterial lipoproteins the N-terminal cysteine of the mature protein is the target of Lnt mediated acylation. Since CexE does not contain features of a typical bacterial lipoprotein it must be recognised and acylated by AatD in a manner different to the acylation of lipoproteins by Lnt.

Further scrutiny of the amino acid sequences of all CexE homologs revealed that for all CexE and Aap proteins the first five amino acids immediately after the signal peptide cleavage site are a conserved mix of glycine and serine residues (Fig 7B). The presence of an invariant N-terminal glycine residue immediately downstream of the predicted signal sequence suggested that this might be the site of acylation. To test this hypothesis, we mutated each of the N-terminal residues of CexE to glutamic acid and observed the effect on acylation by western blotting. The amount of total fluorescence of the secondary antibody bound to primary polyclonal anti-CexE antibody was measured and compared for each mutant. The percentage of acylated mCexE was calculated for each mutant. The migration of the G20E mutant was similar to uCexE indicating that the change to glutamic acid completely abolished acylation (Fig 7C). In addition, the acylation of the G21E mutant was significantly reduced (p-value = 0.0013). However, there was no significant reduction in acylation of the G22E, N23E or S24E mutants 
242 (Fig 7C). These data indicate that the acylation of CexE is highly dependent on the N-terminal glycine 243 at position 20 and to a lesser extent on the second glycine at position 21.

\section{Mass spectrometry analysis of CexE acylation}

Mutation of the conserved N-terminal glycine can abolish acylation of CexE, and Lnt acylates the N-terminal amine of the signal peptidase processed lipoprotein, therefore we hypothesised that AatD would acylate the N-terminal amine of G20. To test this, we first purified his-tagged mCexE and uCexE by nickel affinity chromatography. Both forms of CexE were subjected to trypsin digestion followed by LC-MS/MS. A peak was observed in the HLPC trace of mCexE that was not present in uCexE (Fig 8A). The mass spectrometry of that peptide identified the five N-terminal residues of CexE with an addition of $238 \mathrm{Da}$, which is equal to that expected to a single 16 carbon fatty acid addition (Fig 8B) confirming the modification of CexE by the addition of an acyl chain onto the N-terminal glycine.

\section{Heterologous acylation by AatD}

Other domains of CexE might be responsible for interacting with AatD other than the $\mathrm{N}$ terminus. Therefore, we fused the N-terminus of mCherry with the signal sequence of CexE and an increasing number of glycine residues to a maximum of three. These constructs were transformed into ETEC H10407 $\Delta$ cexE $\mathrm{pCfaD}$ and the production of the mCherry fusions was induced in culture media supplemented with 17-ODYA. The mCherry constructs with $\mathrm{N}$-terminal glycine residues were isolated using the incorporated $\mathrm{C}$-terminal 8xHis tag. The purified proteins were subjected to CuAAC with azide-Cy5. The incorporation of 17-ODYA into the mCherry fusions was observed by detecting fluorescence of the Cy5 dye. The signal sequence alone did not cause an incorporation of 17-ODYA (Fig 9). However, a single glycine at the N-terminus was sufficient for mCherry acylation but the addition of further glycine residues to the $\mathrm{N}$-terminus increased the fluorescence signal indicating an increase of AatD activity proportional with increase in the number of glycine residues present (Fig. 9). 


\section{Discussion}

Post-translational modification of proteins by the covalent attachment of fatty acids occurs for a myriad of proteins in eukaryotic and prokaryotic organisms. Such acylation events confer distinct biochemical properties on the proteins, enabling acylation to regulate intracellular trafficking, subcellular localization, and molecular interactions. The current dogma for Gram-negative bacterial lipoproteins purports that proteins are triacylated on an $\mathrm{N}$-terminal cysteine through the action of three enzymes Lgt, Lsp and Lnt (Grabowicz, 2019; Nakayama et al., 2012; Zückert, 2014). To our knowledge there are no examples of bacterial proteins where acylation occurs on N-terminal glycine residues. While N-terminal glycine acylation by $\mathrm{N}$-myristoyltransferase is present in life it is restricted to eukaryotes. N-myristoylation is one of the three major classes of fatty acylation reactions for eukaryotic proteins, which include: N-myristoylation of glycine/lysine in proteins such as c-Src or HIV Gag1 (Resh, 1994; Veronese et al., 1988); S-palmitoylation of cysteine in proteins such as Ras (Hancock et al., 1989); and N-palmitoylation of cysteine in proteins such as Hedgehog (Pepinsky et al., 1998). Examples of bacterial N-myristoylation rely on eukaryotic proteins produced in E. coli or the injection of myristoylatable proteins into eukaryotic cells by type 3 secretion systems (Duronio et al., 1990; Martin et al., 2011). To our knowledge this is the first report of enzyme mediated N-palmitoylation in nature. The recent publication by Belmont-Monroy et al. 2020 independently identified AatD as an acyltransferase of Aap and CexE.

The Aat system is, to our knowledge, the only secretion system that acylates and secretes a lipoprotein substrate to the cell surface. While surface exposed lipoproteins have been reported for $E$. e.g. Lpp, RcsF, and SslE (Baldi et al., 2012; Cowles et al., 2012; Konovalova et al., 2014), these lipoproteins are acylated by Lgt and Lnt on an N-terminal cysteine in the typical manner and translocated to the outer membrane by the Lol pathway. Similarly, Neisseria spp. decorate their cell surfaces with secreted lipoproteins that are acylated by Lgt and Lnt on an N-terminal cysteine and trafficked to the outer membrane by the Lol system. In the latter case an outer membrane protein termed Slam is required for translocation of the protein to the cell surface (Hooda et al., 2016). In contrast, the Aat system encodes a novel and specialised $\mathrm{N}$-acyltransferase, AatD. To allow secretion of the novel lipoprotein CexE the Aat system has combined proteins of the T1SS and the Lol trafficking pathway. 
Not only does the Aat system secrete a novel class of lipoproteins from the periplasm to the surface of the cell but also it acylates that protein itself. For this reason, we believe that reclassification of the Aat system as the first example of the Lipoprotein Secretion System (LSS) is warranted. We propose that the LSS would remain as a sub-class of the T1SS to emphasise its similarities to the T1SS.

In Fig 10, we propose our model for Aat mediated secretion. First, the Sec machinery secretes preCexE into the periplasm, resulting in cleavage of the signal peptide by Signal Peptidase I to form uCexE (Pilonieta et al., 2007). Once in the periplasm the N-terminal glycine of uCexE is acylated by AatD to create mCexE. Through this acylation event mCexE then associates with the inner membrane. We believe this to be first step for secretion by the Aat system since CexE acylation by AatD is independent of the other members of the Aat system. In addition, the deletion of any of the aatPABC genes does not result in a loss of CexE acylation. We propose that the acylation of CexE is required to enter the AatPABC tunnel. Due to the homology to LolCDE, we believe that mCexE is extracted from the inner membrane by AatPC. Finally, AatA, AatB and AatPC form a channel for mCexE secretion in a manner analogous to a typical T1SS. Subsequently, mCexE inserts into the outer leaflet of the outer membrane.

The Aat system is not unique in secreting a periplasmic substrate. Other T1SS employ a twostep secretion mechanism. One such example is the MacAB-TolC complex which secretes heat-stable enterotoxin from the periplasm (Yamanaka et al., 2008). We identified MacA and MacB as homologs of AatB and AatPC, respectively. Suggesting that AatPC may use a similar mechanism of mechanotransmission as MacB to secrete Aap/CexE (Crow et al., 2017). Furthermore AatB may form a similar gating ring to MacA (Fitzpatrick et al., 2017). However, how the Aat system is able to combine the mechanism of the MacAB-TolC T1SS and the LolCDE lipoprotein trafficking pathway has yet to be elucidated.

Belmont-Monroy et al. 2020 suggested that Lnt is able to acylate Aap. However, in our hands there was no evidence of CexE acylation in the absence of AatD. Given that Aap and CexE lack the traditional lipobox of bacterial lipoproteins that would be recognised by the Lol system, that the signal sequence of all Aap and CexE peptides were predicted to be cleaved by Signal Peptidase I, which has 
acylation in E. coli BL21(DE3) which does not naturally encode AatD or CexE but does encode Lnt, we do not believe that Lnt plays a role in CexE acylation. Indeed, our demonstration of acylation of a heterologous protein with a single glycine residue (Fig 10) suggests that AatD is a specific Nacyltransferase that has the potential for exploitation for production of novel acylated peptides in $E$. coli. The addition of an acyl chain can improve the half-life of peptide drugs like insulin (Kurtzhals, 2007). Since we have shown that it is possible to reconstitute AatD acylation in a laboratory strain of E. coli there are further biotechnological applications to be explored.

Due to increasing levels of antibiotic resistance in pathogenic organisms there is a requirement for new protein targets. The conservation of this system within pathogenic bacterial strains represents a possible new drug target. Previous studies have shown that deletion of aatD, aatC, or aap/cexE reduces bacterial colonisation and disease (Belmont-Monroy et al., 2020; Rivas et al., 2020). Thus, chemicals targeting the Aat system have the potential to prevent or perhaps reduce the severity of bacterial disease. As the Aat system does not appear to be present in non-pathogenic strains this would be an ideal target to prevent off-target effects. As only pathogenic bacteria would be affected by the inhibition of the Aat system, there would be no risk of evolving resistant alleles in non-pathogenic populations.

In conclusion, we have identified a novel $\mathrm{N}$-acyltransferase. In addition, we have characterised a novel class of bacterial lipoproteins that are acylated and secreted by a composite secretion system. There are still significant questions left to be answered on the mechanism of Aat secretion as well as the function of the secreted proteins. In addition, the ability to acylate heterologous proteins with a simple glycine addition is a valuable new tool for the biotechnology enabling new acylated peptides to be produced in E. coli. 


\section{Materials and methods}

\section{Bacterial cultivation}

Supplementary Table S3 contains the bacterial strains used in this study. Bacterial strains were regularly cultivated in lysogeny broth (LB) which consisted of $10 \mathrm{~g} / \mathrm{L}$ tryptone, $5 \mathrm{~g} / \mathrm{L}$ yeast extract and $10 \mathrm{~g} / \mathrm{L}$ $\mathrm{NaCl}$. Bacterial strains were routinely grown on LB supplemented with $1.25 \%$ agar (LBA), and a single colony was used to inoculate liquid cultures for overnight growth at $37^{\circ} \mathrm{C}$ with aeration. Overnight cultures were used to inoculate Erlenmeyer flasks containing a fifth of their total volume of LB supplemented with the relevant antibiotics, and grown at $37^{\circ} \mathrm{C}$ with aeration. Antibiotics were used at the following final concentrations: kanamycin $100 \mu \mathrm{g} / \mathrm{ml}$; carbenicillin $100 \mu \mathrm{g} / \mathrm{ml}$; and chloramphenicol $35 \mu \mathrm{g} / \mathrm{ml}$. For the induction of CexE in ETEC H10407, cultures harbouring pCfaD were grown for 90 minutes in $\mathrm{LB}$, supplemented with carbenicillin, at $37^{\circ} \mathrm{C}$ with aeration. Expression of $c f a D$ was induced with $0.2 \%$ L-arabinose for 2 hours at $37^{\circ} \mathrm{C}$ with aeration. Aap was induced by growing EAEC 042 in DMEM-HG with aeration at $37^{\circ} \mathrm{C}$.

\section{Molecular techniques}

Primers for PCR can be found in Table S4. Plasmids used in this study are detailed in Table S5. Unless otherwise stated all primers were used at a concertation of $10 \mu \mathrm{M}$. Q5® High-Fidelity 2 X Master Mix (NEB) was used for cloning or mutagenesis. MyTaq ${ }^{\mathrm{TM}}$ Red Mix (Bioline) was used in all other cases. The cexE gene from ETEC H10407 was amplified using CcexE-F and CcexE-R primers. Both the vector, pET26b(+) (Novagen), and the insert were cut with NdeI (NEB) and XhoI (NEB) using the CutSmart ${ }^{\circledR}$ (NEB) protocol. The digested vector was treated with Antarctic Phosphatase (NEB). T4 DNA Ligase (NEB) was used to ligate the insert DNA to the vector DNA. The ligation mixture was transformed into NEB® ${ }^{\circledR}$-alpha Competent E. coli (High Efficiency). Recovered cells were plated on LBA supplemented with $100 \mu \mathrm{g} / \mathrm{ml}$ kanamycin and incubated at $37^{\circ} \mathrm{C}$ overnight. The cexE gene and 573 bp upstream of the cexE gene, which included the two CfaD binding sites (Pilonieta et al., 2007), was amplified by PCR. The reverse primer included a 6 His tag. The resulting amplified DNA was digested with SphI and BamHI and ligated into pACYC184. Successful ligations were selected for as 

constructed using the QuickChange II method (Aglient). The aat genes and cexE were disrupted in ETEC H10407 as previously described (Datsenko \& Wanner, 2000). The plasmid pDOC-K was used as the source of the kanamycin resistance cassette (Lee et al., 2009). The cexE-mCherry fusion and the aatD gene from ETEC H10407 were synthesised by GenScript. Deletions of the cexE gene from the plasmid encoding the CexE-mCherry fusion were constructed as previously described (Moore \& Prevelige, 2002). Plasmid sequences were confirmed by Sanger sequencing.

\section{Bioinformatic analysis of the Aat system}

The protein sequences of the Aat proteins from ETEC H10407 were used to search the NCBI nonredundant protein sequences database using PSI-BLAST (Altschul et al., 1997). Strains encoding a complete Aat system on the same nucleotide accession were used to assess the distance between aat genes. Distant homologs of the Aat proteins were identified using HMMER (Finn et al., 2011). An HMM was created for each Aat protein using the Aat protein sequences identified by PSI-BLAST.

These models were used to search the UniprotKB or Swissprot databases (The UniProt Consortium, 2019). Protein sequences were aligned using Clustal Omega (Madeira et al., 2019). RAxML was used for the construction of phylogenetic trees (Stamatakis, 2014). Trees were drawn using iTOL (Letunic \& Bork, 2019).

\section{CexE purification for antibody production}

BL21(DE3) was transformed with pET26b-cexE. An overnight culture of BL21(DE3) pET26b-cexE was used to inoculate $2 \mathrm{~L}$ of $\mathrm{LB}$ supplemented with $100 \mu \mathrm{g} / \mathrm{ml}$ kanamycin. The culture was grown at $37^{\circ} \mathrm{C}$ with aeration to an $\mathrm{OD}_{600}$ of 0.4 . The culture was moved to $20^{\circ} \mathrm{C}$ incubator for 30 min prior to induction. CexE production was induced with $50 \mu \mathrm{M}$ Isopropyl $\beta$-D-1-thiogalactopyranoside (IPTG) (Sigma) and incubated overnight at $20^{\circ} \mathrm{C}$ with aeration. After overnight growth, cells were harvested by centrifugation at $6,000 \times \mathrm{g}$ for $10 \mathrm{~min}$ at $4^{\circ} \mathrm{C}$. The cell pellet was resuspended in ice-cold binding buffer $\left(50 \mathrm{mM} \mathrm{NaP}\right.$ (77:33 ratio of $\mathrm{Na}_{2} \mathrm{HPO}_{4}$ to $\left.\mathrm{NaH}_{2} \mathrm{PO}_{4}\right), 500 \mathrm{mM} \mathrm{NaCl}, 20 \mathrm{mM}$ imidazole, $0.5 \mathrm{mM}$ 
were removed by centrifugation at $10,000 \times \mathrm{g}$ for $10 \mathrm{~min}$ at $4^{\circ} \mathrm{C}$. The cellular membrane components were removed by centrifugation at $75,000 \times g$ for $1 \mathrm{~h}$ at $10^{\circ} \mathrm{C}$. The supernatant was applied to a HisTrap ${ }^{\text {TM }}$ HP $5 \mathrm{ml}$ column (GE Healthcare Life Sciences) overnight at $4{ }^{\circ} \mathrm{C}$. The column was washed with 5 column volumes of binding buffer then the protein was eluted in $5 \mathrm{ml}$ fractions with elution buffer (50 mM NaP, $500 \mathrm{mM} \mathrm{NaCl}, 500 \mathrm{mM}$ imidazole, $0.5 \mathrm{mM}$ TCEP at $\mathrm{pH}$ 7.3). Samples containing purified protein were concentrated using Vivaspin® 6 5,000 MWCO columns (Sartorius Stedim). Protein was buffer exchanged using membrane filtration into $50 \mathrm{mM} \mathrm{NaP}, 250 \mathrm{mM} \mathrm{NaCl}$ and $0.5 \mathrm{mM}$ TCEP at $\mathrm{pH}$ 7.3. Purified CexE protein was used to produce primary antibodies by Eurogentec using the 28-day speedy protocol. Polyclonal antibodies against CexE were concentrated prior to use for the detection of CexE.

\section{Tris-Tricine SDS-PAGE and western blotting}

Protein samples were separated using tris-tricine SDS-PAGE as previously described (Schägger, 2006).

In brief, protein samples were mixed with sample buffer (4\% SDS, 2.5\% 2-mercaptoethanol, 7.5\% glycerol, $0.01 \%$ bromophenol blue, $35 \mathrm{mM}$ Tris $(\mathrm{pH} 7.0)$ ) and separated on 10\% tris-tricine SDS-PAGE gels. Gels were stained with Coomassie brilliant blue or transferred to nitrocellulose for western blotting. Nitrocellulose membranes were covered in 2\% BSA (20 g BSA, 2.42 g Tris-base (pH 8.4), 8 $\mathrm{g} \mathrm{NaCl}$ per L). The primary antibody was diluted in $2 \% \mathrm{BSA}$ at the following concentrations: Aap 1 in 5,000 (Sheikh et al., 2002); CexE 1 in 2,000 (this study); and $\beta$ subunit of RNAP 1 in 10,000 (E. coli RNA Polymerase beta Monoclonal Antibody, BioLegend). Primary antibodies were incubated overnight at $4^{\circ} \mathrm{C}$ with agitation. Membranes were washed three times in TBST (2.42 g Tris-base, $8 \mathrm{~g}$ $\mathrm{NaCl}, 0.1 \%$ Tween-20, $\mathrm{pH} 8.4$ in $1 \mathrm{~L}$ ) for $5 \mathrm{~min}$ at room temperature with agitation prior to incubation with secondary antibody. Anti-Rabbit (IRDye 800CW Goat anti-Rabbit IgG, Li-Cor) or anti-mouse (IRDye 680LT Goat anti-Mouse IgG, Li-Cor) secondary antibodies were used to detect primary antibody binding. Secondary antibodies were diluted 1 in 15,000 in $2 \%$ BSA and incubated for a minimum of $1 \mathrm{~h}$ at room temperature with agitation. Membranes were washed 4 times with TBST for $5 \mathrm{~min}$ at room temperature with agitation. Secondary antibody fluorescence was detected using the Odessy CLx imaging system. 


\section{Inhibition of SecA by sodium azide}

427 Two cultures of ETEC H10407 pCfaD were inoculated from the same overnight culture. The cells were both grown at $37^{\circ} \mathrm{C}$ for 90 min with aeration. WCL samples of each culture were taken. L-arabinose was added to both cultures to a final concentration of $0.2 \%$. Sodium azide was added to a final concentration of $3 \mathrm{mM}$ to one of the cultures. Both cultures were grown for $2 \mathrm{~h}$ at $37^{\circ} \mathrm{C}$ with aeration. WCL samples of each culture were taken and separated by tris-tricine SDS-PAGE. CexE was detected by western blotting.

\section{Proteomic analysis}

Cultures were grown under the conditions previously described for CexE production in ETEC H10407 $\mathrm{pCfaD}$. Cells were separated from the culture supernatant by centrifugation at $8,000 \times \mathrm{g}$ for $10 \mathrm{~min}$ at $4^{\circ} \mathrm{C}$. The culture supernatant was filtered using Millex-GP Syringe Filter Unit, $0.22 \mu \mathrm{m}$, polyethersulfone (Merck) then cooled on ice. Ice-cold 100\% TCA was added to a final concentration of $20 \%$. Samples were incubated on ice for 30 minutes. Proteins were pelleted by centrifugation. The supernatant was removed and discarded from the sample. The pellet was washed twice with $1 \mathrm{ml}$ icecold $100 \%$ methanol, proteins were collected with centrifugation at $21,000 \mathrm{x} g$ for $15 \mathrm{~min}$ at $4^{\circ} \mathrm{C}$ between each wash. The supernatant was removed and residual methanol was evaporated by incubating the sample at $60^{\circ} \mathrm{C}$ for $10 \mathrm{~min}$. The pellet was resuspended in $50 \mu \mathrm{l}$ tris-tricine sample buffer. If a colour change to yellow occurred saturated tris-base was added until the original colour returned. Samples were analysed by tris-tricine SDS-PAGE.

Membranes were extracted from $50 \mathrm{ml}$ of cells grown under the conditions required for CexE production. Cells were collected by centrifugation and resuspended in $20 \mathrm{ml}$ of $10 \mathrm{mM}$ Tris (pH 8.0), 1 mM PMSF (Phenylmethylsulfonyl fluoride). Cells were lysed using EmulsiFlex®-C3 (Avestin). Unlysed cells were collected by centrifugation at $5,000 \mathrm{xg}$ for 10 minutes at $4^{\circ} \mathrm{C}$. The supernatant was separated. The membranes were collected by centrifugation at $50,000 \mathrm{x}$ g for 60 minutes at $4^{\circ} \mathrm{C}$. The resulting membrane pellet was washed twice with ice-cold $10 \mathrm{mM}$ Tris $(\mathrm{pH} \mathrm{8.0)}$. The final pellet was resuspended in $100 \mu 10 \mathrm{mM}$ Tris ( $\mathrm{pH}$ 8.0). The samples were normalised by protein concentration. 
Overnight cultures harbouring plasmids encoding his tagged proteins of interest to be labelled were used to inoculate $25 \mathrm{ml} \mathrm{LB}$ supplemented with the relevant antibiotics to an $\mathrm{OD}_{600}$ of 0.05 . Cultures were grown for $1.5 \mathrm{~h}$ at $37^{\circ} \mathrm{C}$. 17-ODYA dissolved in DMSO was added to a final concentration of 20 $\mu \mathrm{M}$ and protein production induced. An equal volume of DMSO was added as a negative control. Cells were collected by centrifugation and resuspended in $600 \mu 1$ of $50 \mathrm{mM} \mathrm{NaP}(\mathrm{pH} 7.0), 150 \mathrm{mM} \mathrm{NaCl}$, $0.1 \%$ Triton X-100, and $1 \mathrm{mM}$ PMSF. Cells were lysed by sonication for $15 \mathrm{~min}$ using the bioruptor on a $30 \mathrm{~s}$ on, $30 \mathrm{~s}$ off cycles. Insoluble material was removed by centrifugation at and the supernatant was retained. His tagged proteins were isolated from the supernatant using Dynabeads ${ }^{\mathrm{TM}}$ His-Tag Isolation and Pulldown (Invitrogen) as per the manufacturer's instructions. Imidazole was removed by dialysis. Protein sample concentrations were measured with Pierce ${ }^{\mathrm{TM}}$ BCA Protein Assay Kit (Thermo Scientific $^{\mathrm{TM}}$ ). A master mix was prepared of $0.2 \mathrm{mM}$ Cy5-azide (Sigma-Aldrich) or Alexa Fluor ${ }^{\mathrm{TM}} 488$ azide (Invitrogen), $0.2 \mathrm{mM}$ tris[(1-benzyl-1H-1,2,3-triazol-4-yl)methyl]amine (TBTA), and $2 \mathrm{mM}$ freshly prepared $\mathrm{CuSO}_{4}$. This was made to $50 \mu \mathrm{l}$ in $\mathrm{H}_{2} \mathrm{O}$ and $5 \mu \mathrm{l}$ of the master mix was added to $40 \mu \mathrm{l}$ each normalised protein sample. Sodium ascorbate was added to a final concentration of $5 \mathrm{mM}$. Samples were incubated at $37^{\circ} \mathrm{C}$ for $1 \mathrm{~h}$ with agitation. Protein was precipitated using chloroform-methanol extraction. To each sample $400 \mu \mathrm{l} \mathrm{MeOH}, 150 \mu \mathrm{l}$ chloroform, and $300 \mu 1 \mathrm{H}_{2} \mathrm{O}$ were added and vortexed for 30 seconds. Samples were centrifuged at max speed for $2 \mathrm{~min}$. The top layer was removed and 400 $\mu \mathrm{MeOH}$ was added and samples were centrifuged again at max speed for 2 min. The supernatant was removed, and the pellet was washed twice with $400 \mu \mathrm{MeOH}$ then dried. Pellets were resuspended in $20 \mu 11 \mathrm{x}$ tris-tricine sample buffer and separated by tris-tricine SDS-PAGE. Gels were incubated in

474 fixative (10\% acetic acid, 50\% methanol) prior to fluorescence detection then stained with Coomassie Brilliant Blue.

\section{Heterologous protein acylation}

477 ETEC H10407 pCfaD was transformed with pRSF-GGG-mCherry, pRSF-GG-mCherry, pRSF-G478 mCherry or pRSF-SS-mCherry. Cultures were grown as previously described for $c f a D$ induction. At the same time as $c f a D$ was induced 17-ODYA was added to a final concentration of $20 \mu \mathrm{M}$. Cells were 
grown for 2 hours and harvested by centrifugation. 17-ODYA incorporation was detected as described

481 above.

\section{Mass spectrometry analysis of CexE acylation}

CexE with a 6 His tag was isolated from a cexE mutant and a cexE aatD double mutant of ETEC H10407

pCfaD. The isolated CexE proteins were separated on a tris-tricine gel and stain with Coomassie brilliant blue. The band corresponding to $\mathrm{uCexE}$ and mCexE were excised and subjected to trypsinLysC digestion. The tryptic peptide extracts were analyzed by nanoHPLC/MS MS/MS on an Eksigent, acetonitrile. Linear gradients of 2-40\% solvent B over $60 \mathrm{~min}$ at $400 \mathrm{~nL} /$ minute flow rate, followed by a steeper gradient from $40 \%$ to $90 \%$ solvent B in $5 \mathrm{~min}$, then $90 \%$ solvent B for $5 \mathrm{~min}$, were used for peptide elution. The gradient was then returned to $2 \%$ solvent $\mathrm{B}$ for equilibration prior to the next sample injection. The ionspray voltage was set to $2600 \mathrm{~V}$, declustering potential (DP) $80 \mathrm{~V}$, curtain gas flow 30, nebuliser gas 1 (GS1) 30, interface heater at $150^{\circ} \mathrm{C}$. The mass spectrometer acquired $50 \mathrm{~ms}$ full scan TOF-MS data followed by up to $30100 \mathrm{~ms}$ full scan product ion data, with a rolling collision energy, in an Information Dependant Acquisition, IDA, mode for protein identification and peptide library production. Full scan TOF-MS data was acquired over the mass range 350-1800 and for product ion MS/MS 100-1500. Ions observed in the TOF-MS scan exceeding a threshold of 200 counts and a charge state of +2 to +5 were set to trigger the acquisition of product ion, MS/MS spectra of the resultant

50330 most intense ions. The data was acquired and processed using Analyst TF 1.7 software (ABSCIEX,

504 Canada). Protein identification was carried out using Protein Pilot 5.0 for database searching. 


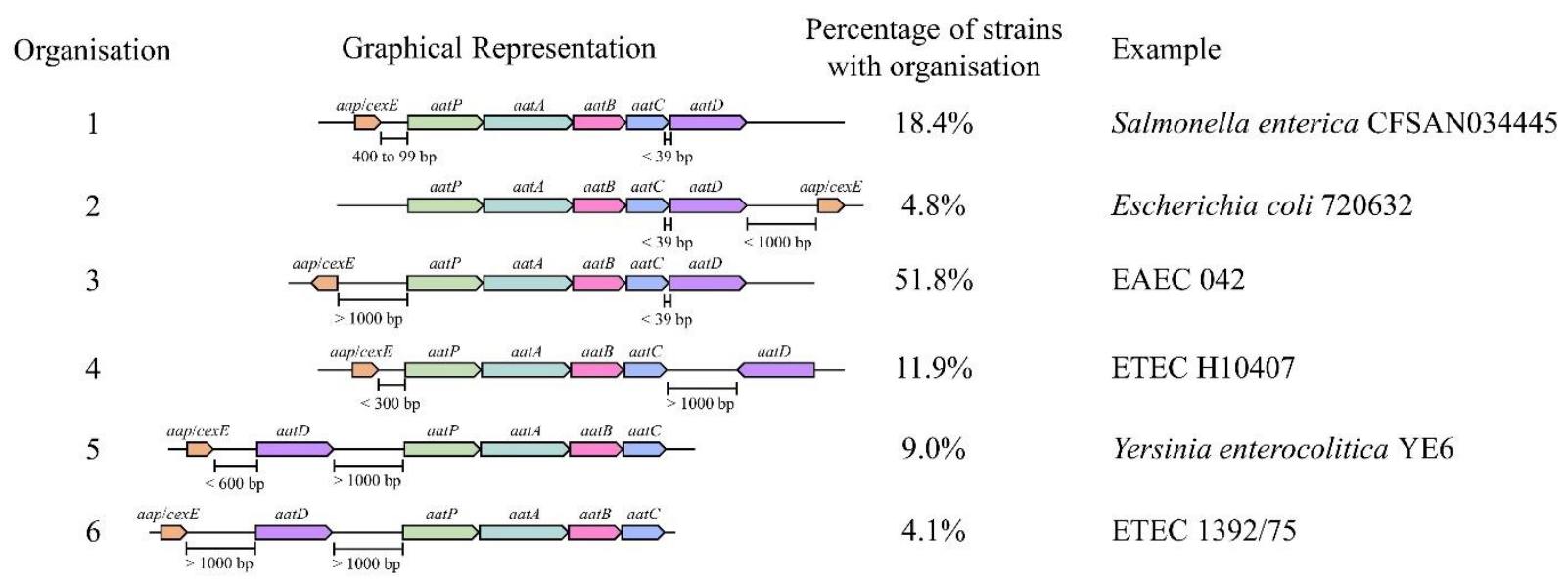

507 Fig 1. Organisation of the Aat operon. Unique Aat amino acid sequences were detected using PSI-

508 BLAST. These sequences were using to identify the strains that encoded them in the NCBI identical

509 protein groups. As the aat genes were present on contigs of whole genome sequencing projects it was

510 not possible to assess if a strain encoded a gene on the chromosome or plasmid. Instead contigs were

511 used to identify complete the complete Aat system. This analysis does not include strains that might

512 encode the aat genes or aap/cexE on a separate genomic element. However, a total of 827 complete Aat

513 systems were identified in the same nucleotide accession. The positions of these genes were used to

514 assess the organisation of the aat operon. From this assessment five different classes of aat operon

515 organisation were defined. 

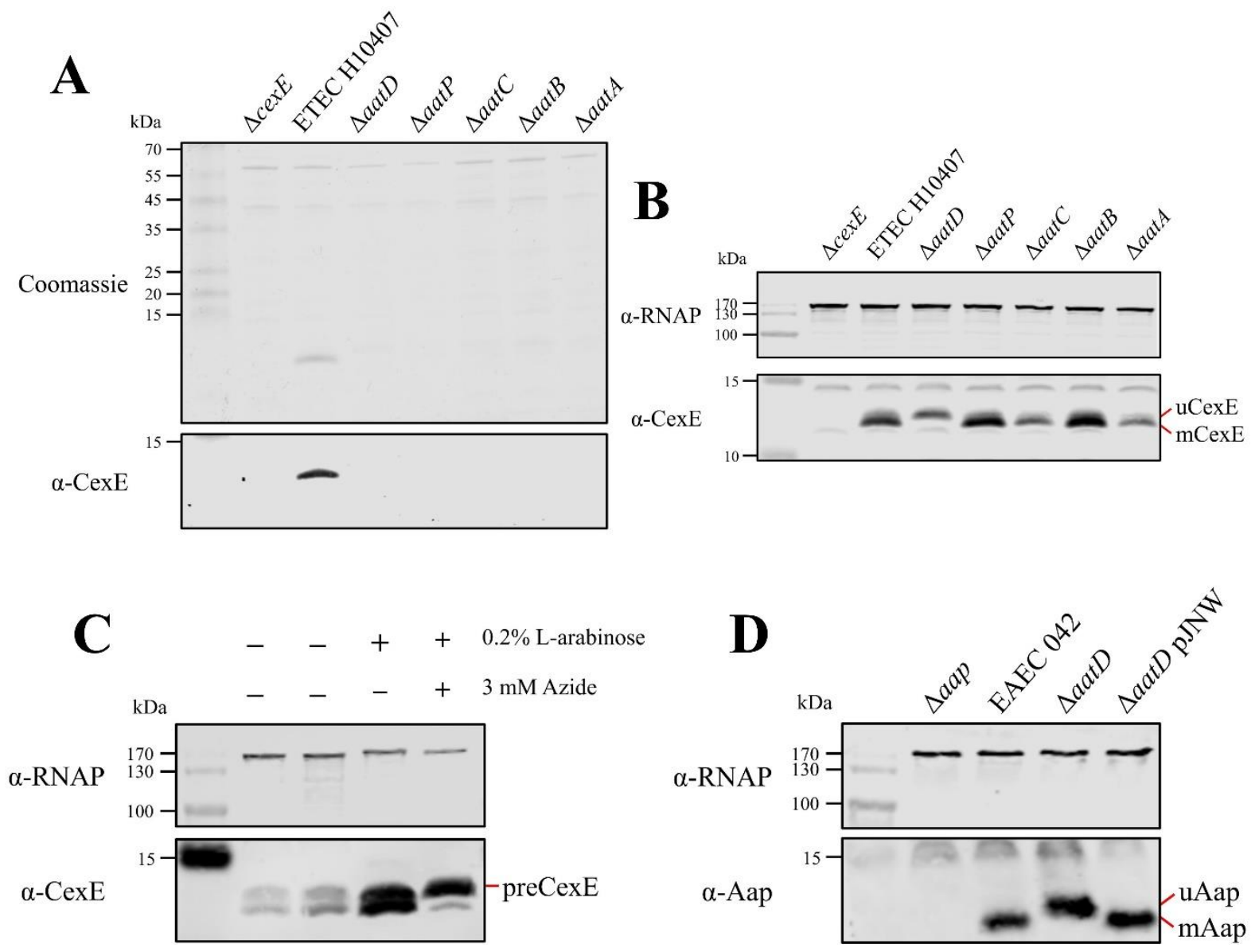

517 Fig 2. AatD post-translational modification of Aap and CexE. (A) Culture supernatant of ETEC

518 H10407 and aat mutants harbouring pCfaD grown in LB supplemented with L-arabinose. Cells were 519 removed from the culture supernatant and remaining protein precipitated with trichloroacetic acid. 520 Protein samples were separated by tris-tricine SDS-PAGE and stained with Coomassie or transferred to nitrocellulose for western blotting with polyclonal antibodies against CexE. (B) Whole cell lysates of the aat mutants and parental strain separated by tris-tricine SDS-PAGE. (C) Whole cell lysates of ETEC H10407 grown in LB with or without azide. (D) Whole cell lysates of EAEC 042, aap, aatD and aat $D$ complemented strains grown in DMEM high glucose. The positions of uCexE, mCexE, uAap, mAap, and preCexE are indicated as appropriate. CexE and Aap were detected by western blotting ( $\alpha$ -

526 CexE and $\alpha$-Aap, respectively) and RNA polymerase ( $\alpha$-RNAP) was included as a loading control 527 where appropriate. 


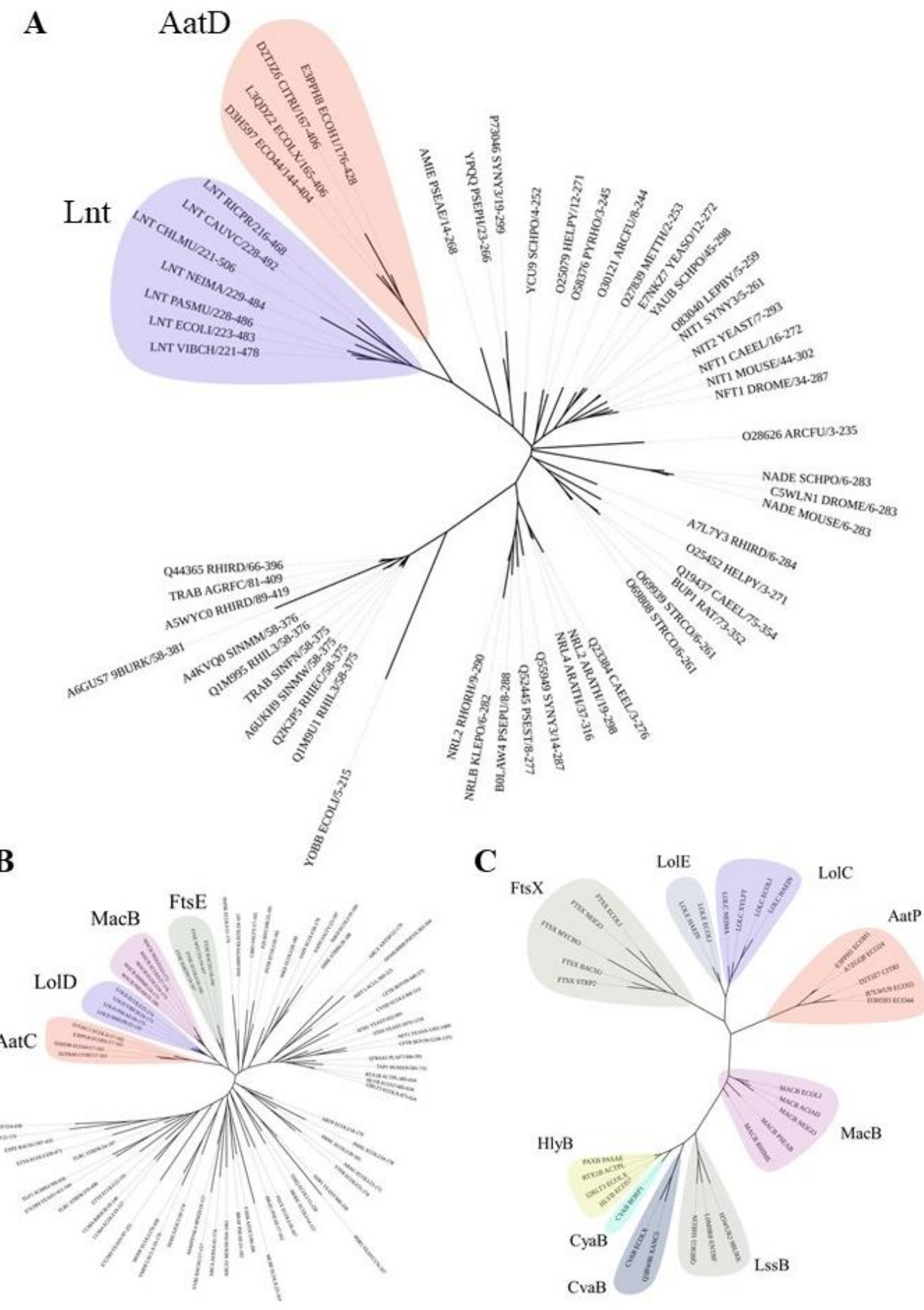

530 Fig 3. Phylogenetic analysis of AatD, AatP and AatC. Sequences were aligned using Clustal omega

531 and the tree was calculated using RAxML. (A) The C-N hydrolase domains of C-N hydrolase family

532 seed sequences of the were aligned to the C-N hydrolase domains of AatD sequences from ETEC

533 H10407, EAEC 042, C. rodentium ICC168, and E. coli KTE75. (B) Phylogram of the ATPase domains

534 of the pfam ABC transporters (PF00005) and AatC sequences. (C) Phylogram of T1SS ABC transporters, $\mathrm{Lol} \mathrm{ABC}$ transporters and AatP sequences. 

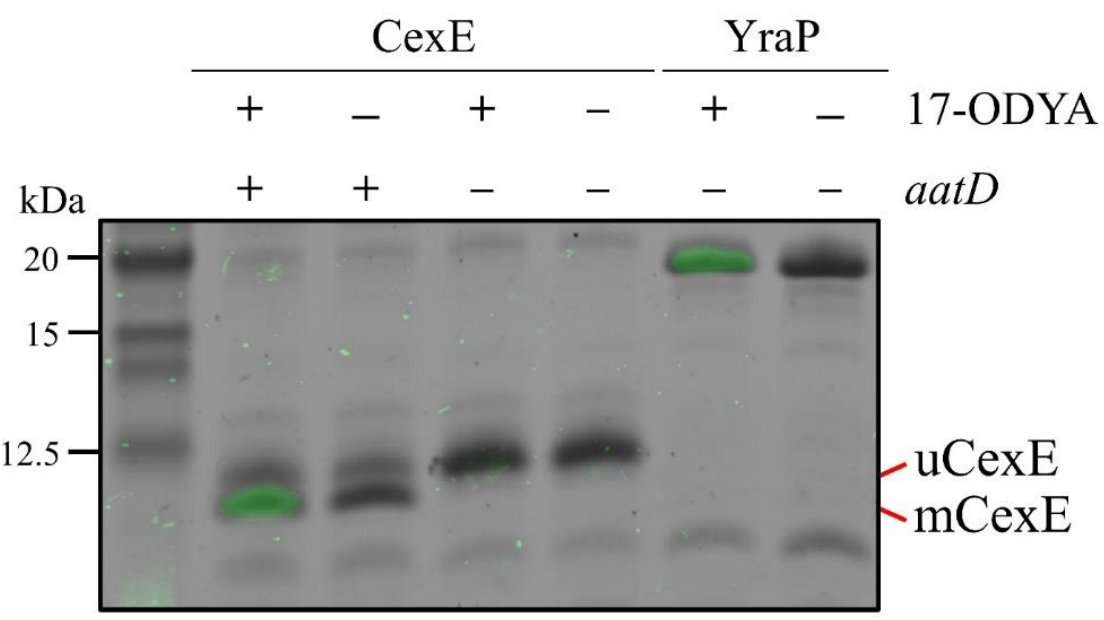

537 Fig 4. Incorporation of 17-ODYA into CexE in the presence of AatD. His tagged CexE was isolated

538 from cexE or cexE aatD double mutants harbouring pACYC-cexE-6His grown in the presence or 539 absence of 17-ODYA and separated by SDS-PAGE. An azide linked Alexa Fluor 488 was conjugated 540 to the alkyne moiety present in 17-ODYA by CuAAC. The incorporation of 17-ODYA into the target

541 protein was detected by fluorescence (green bands) and the image was overlaid on the image of the

542 SDS-PAGE gel. A known lipoprotein YraP was used as a positive control. 


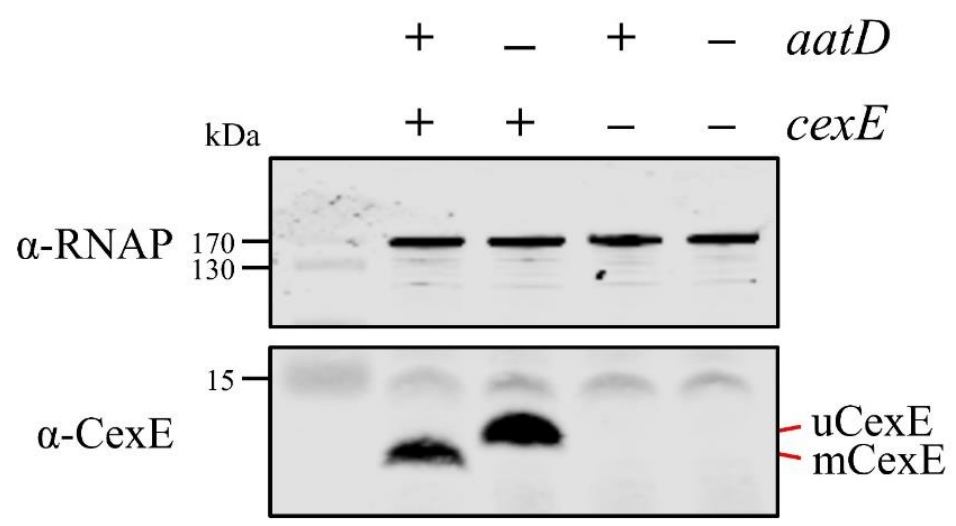

545 Fig 5. Recapitulation of CexE acylation in $\boldsymbol{E}$. coli BL21(DE3). E. coli BL21(DE3) was transformed 546 with pET26b, or pET26b-cexE and pACYCDuet-1 or pACYC-aatD. Cultures were grown in LB and 547 protein production was stimulated with IPTG. Whole cell lysate samples were taken and separated by 548 tris-tricine SDS-PAGE. CexE was detected using a polyclonal antibody. RNAP was used as a loading control. The position of mCexE and uCexE are indicated. 
A

\section{Lnt_MG1655} AatD_H10407
AatD_042 LI IWPESAITDLEINQQPFLKALDGELRDKGSSLVTGIVDARLNKQN--------RYDTYN
MVVFSENTVYGFKN---QEN-------KKITQKMISDLKASNAHQRHAFIFNFFGFNDIN MVVESENTVYGEKN---QFN-------KKI TQKMISDLKASNAHQRHAFIFNFFGFNDIN
LIILSENVFFGYKN---DYI------KERTKHLLKQLKDNRLHYYGI LMNLYGYQDIN

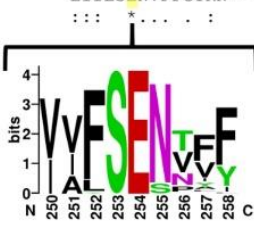

Lnt MG1655 AatD_H10407

TIITLGKGAPYSYESADRYNKNHLVPFGEFVPLESILRPLAPFFDLPMSSFSRGPYIQPP TIITLGKGAPYSYESADRYNKNHLVPFGEFVPLESI LRP LAPFF DLPMSSFSRGPYIQP NVVS----AFWHKEEFLIHQKSKLIPFFEKKSFYNSPEPST----SPFLYYKKKYNEQD NVVS- $:::$

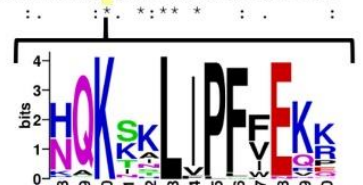

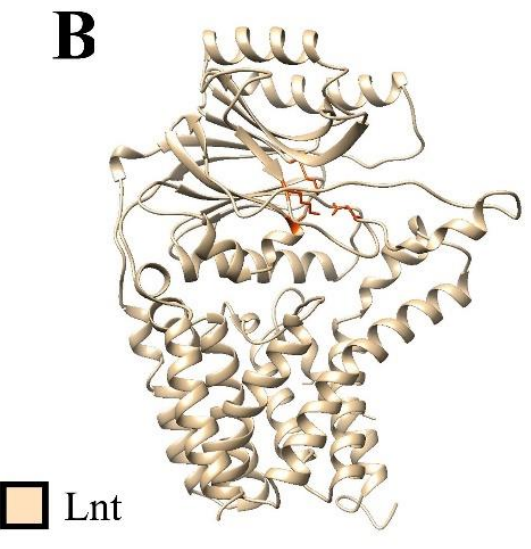

$\square$ AatD

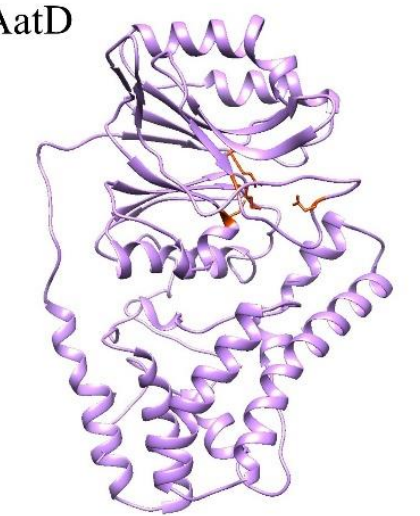

C

SANGIELTAAICYEITLGEQVRDNERPDTDYLLTISNDAWFGKSIGPWQHFQ----MAR INHNGINIKTYICYDVLFPETHKS-----DNELVIVQSNYKLIDKNDMYNRI I KNGSILG DFNNI KMS IH ICYEGLFPEGESR----RKDISIVQSDYSWLSDNHKY DNTL INGSILS Lnt_MG1655 AatD_042
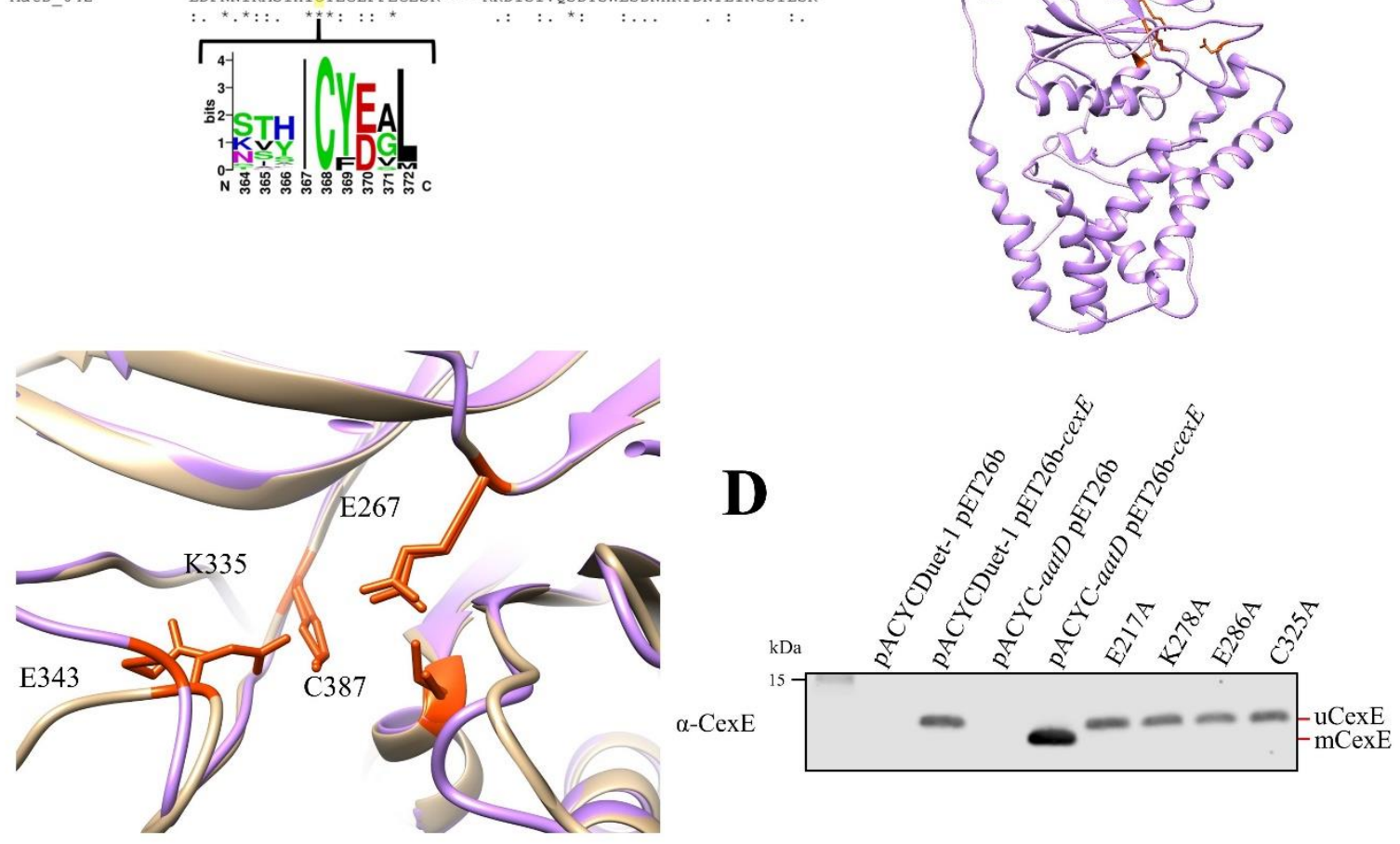

Fig 6. Catalytic residues of AatD. (A) AatD from EAEC 042 and ETEC H10407 were aligned with Lnt from MG1655. AatD sequences identified by PSI-BLAST were aligned and used to create a Weblogo. The catalytic residues of the C-N hydrolase family are highlighted. (B) The structure of Lnt compared to the predicted structure of ETEC H10407 AatD. The catalytic residues of Lnt and AatD are highlighted in orange. (C) Magnified view of the catalytic site of Lnt with the predicted structure of AatD superimposed. (D) Residues are numbered as they appear in Lnt. E. coli BL21(DE3) was producing CexE in the presence of each of the four single site substitution mutants of AatD. CexE was detected using a polyclonal antibody. 


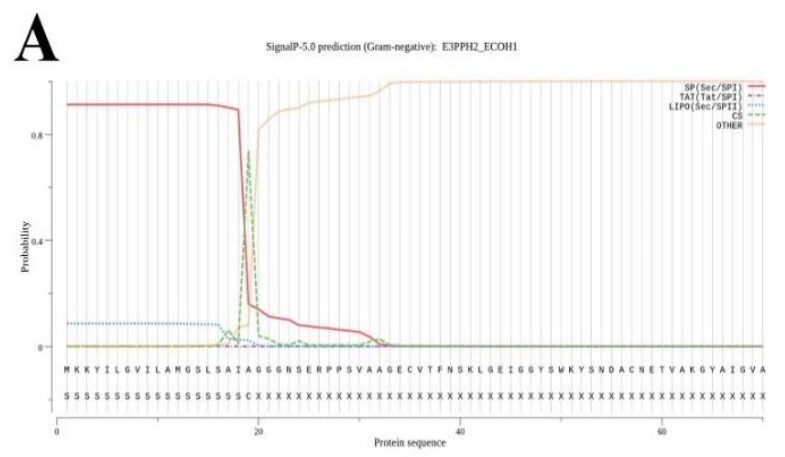

\section{B}
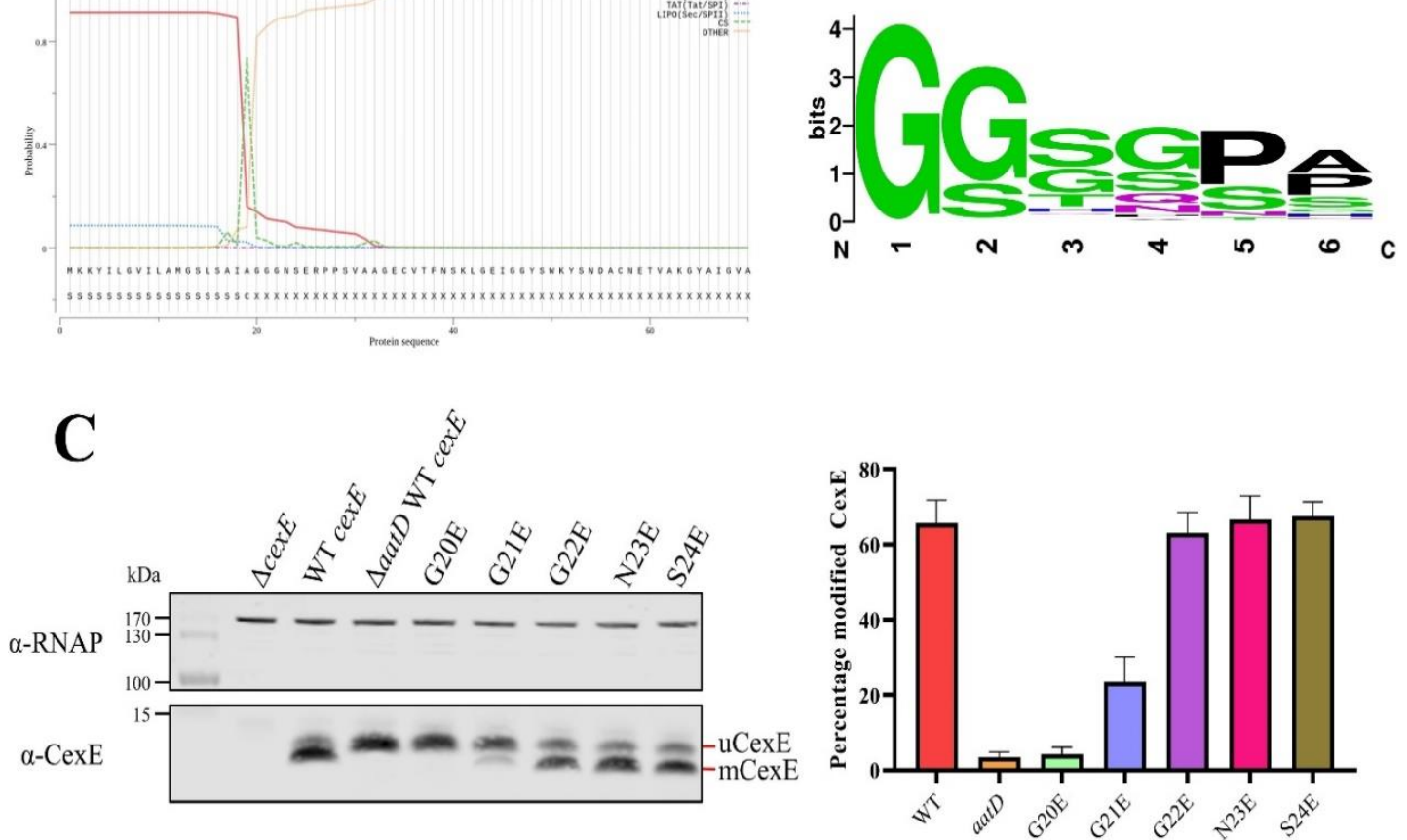

561 Fig 7. Single site substitution of the 5 N-terminal amino acids of CexE. (A) SignalP result of CexE sequence from ETEC H10407 (E3PPH2_ECOH1) (B) Weblogo of the 5 N-terminal residues of Aap and CexE sequences post Sec signal sequence cleavage. (C) ETEC H10407 cexE mutants transformed

564 with pACYC184 ( $\Delta c e x E)$ or pACYC-cexE-6His with either the wild-type sequence (WT cexE) or one

565 of the first five amino acids mutated to glutamic acid. CexE was detected by polyclonal antibodies and 566 RNAP was used as a loading control. The percentage of mCexE was determined from 3 biological replicates. 

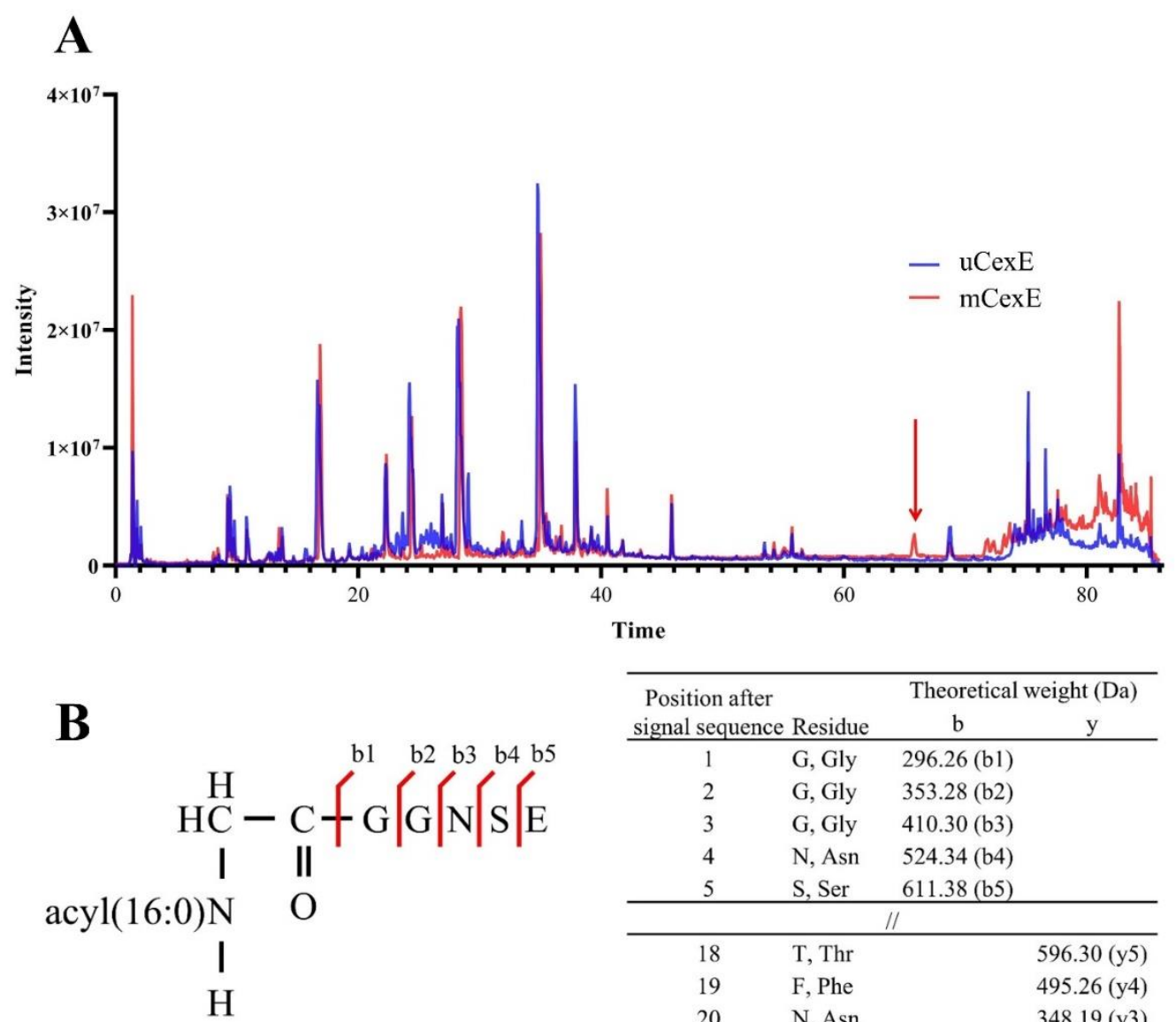

\begin{tabular}{|c|c|c|c|}
\hline \multirow{2}{*}{\multicolumn{2}{|c|}{$\begin{array}{l}\text { Position after } \\
\text { signal sequence Residue }\end{array}$}} & \multicolumn{2}{|c|}{ Theoretical weight (Da) } \\
\hline & & $\mathrm{b}$ & $\mathrm{y}$ \\
\hline 1 & G, Gly & 296.26 (b1) & \\
\hline 2 & G, Gly & $353.28(\mathrm{~b} 2)$ & \\
\hline 3 & G, Gly & $410.30(\mathrm{~b} 3)$ & \\
\hline 4 & $\mathrm{~N}$, Asn & $524.34(\mathrm{~b} 4)$ & \\
\hline 5 & S, Ser & $611.38(\mathrm{~b} 5)$ & \\
\hline \multicolumn{4}{|c|}{11} \\
\hline 18 & $\mathrm{~T}, \mathrm{Thr}$ & & $596.30(\mathrm{y} 5)$ \\
\hline 19 & F, Phe & & $495.26(\mathrm{y} 4)$ \\
\hline 20 & $\mathrm{~N}$, Asn & & $348.19(\mathrm{y} 3)$ \\
\hline 21 & S, Ser & & $234.14(\mathrm{y} 2)$ \\
\hline 22 & $\mathrm{~K}$, Lys & & $147.11(\mathrm{yl})$ \\
\hline
\end{tabular}

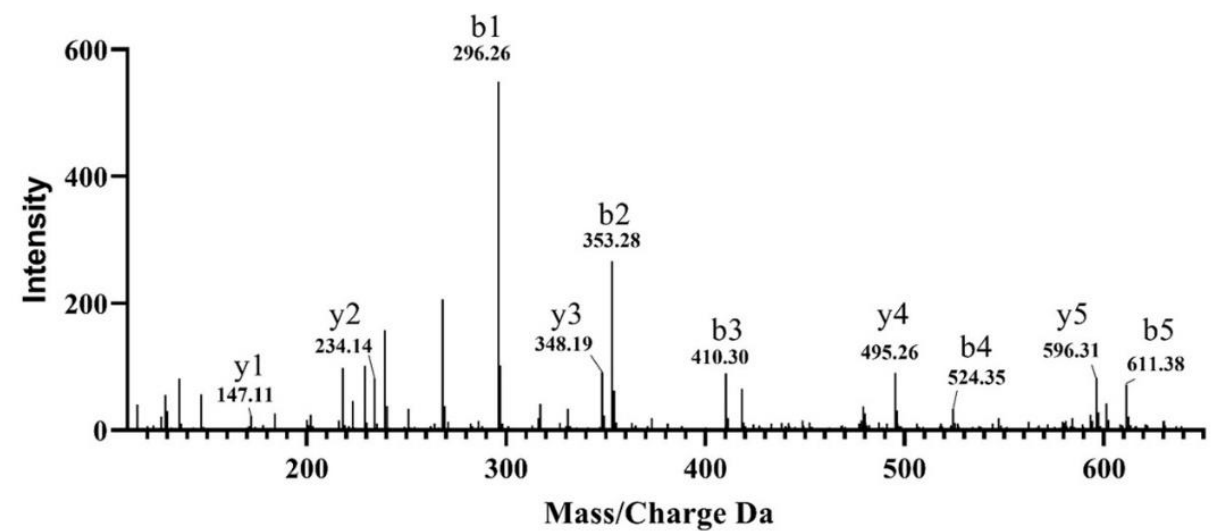

\section{Mass/Charge Da}

569 Fig 8. Mass spectrometry analysis of modified CexE. (A) CexE-6His was isolated from a cexE mutant

570 and a cexE aatD double mutant and subjected to LC-MS/MS. CexE and pro-CexE were trypsinated and

571 separated by HPLC. (B) The indicated peak was subjected to MS/MS to identify the amino acid

572 sequence. 


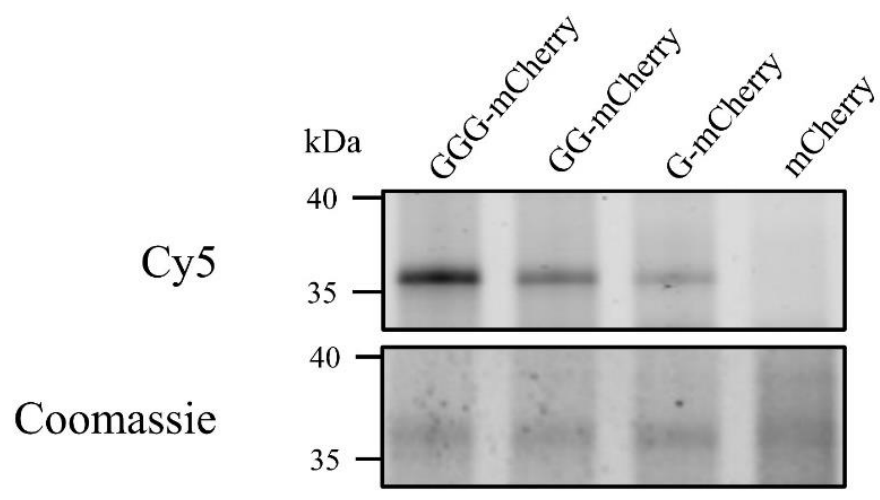

575 Fig 9. Heterologous acylation by AatD plasmid encoding mCherry with the CexE signal sequence

576 followed by none, one (G), two (GG) or three (GGG) glycine residues post signal sequence was

577 produced in an ETEC H10407 cexE mutant grown in the presence of 17-ODYA. The mCherry proteins

578 were isolated using a C-terminal 8 His tag and azide linked Cy5 was incorporated into mCherry proteins

579 using CuAAC. The acylation of mCherry was detected using fluorescence. 


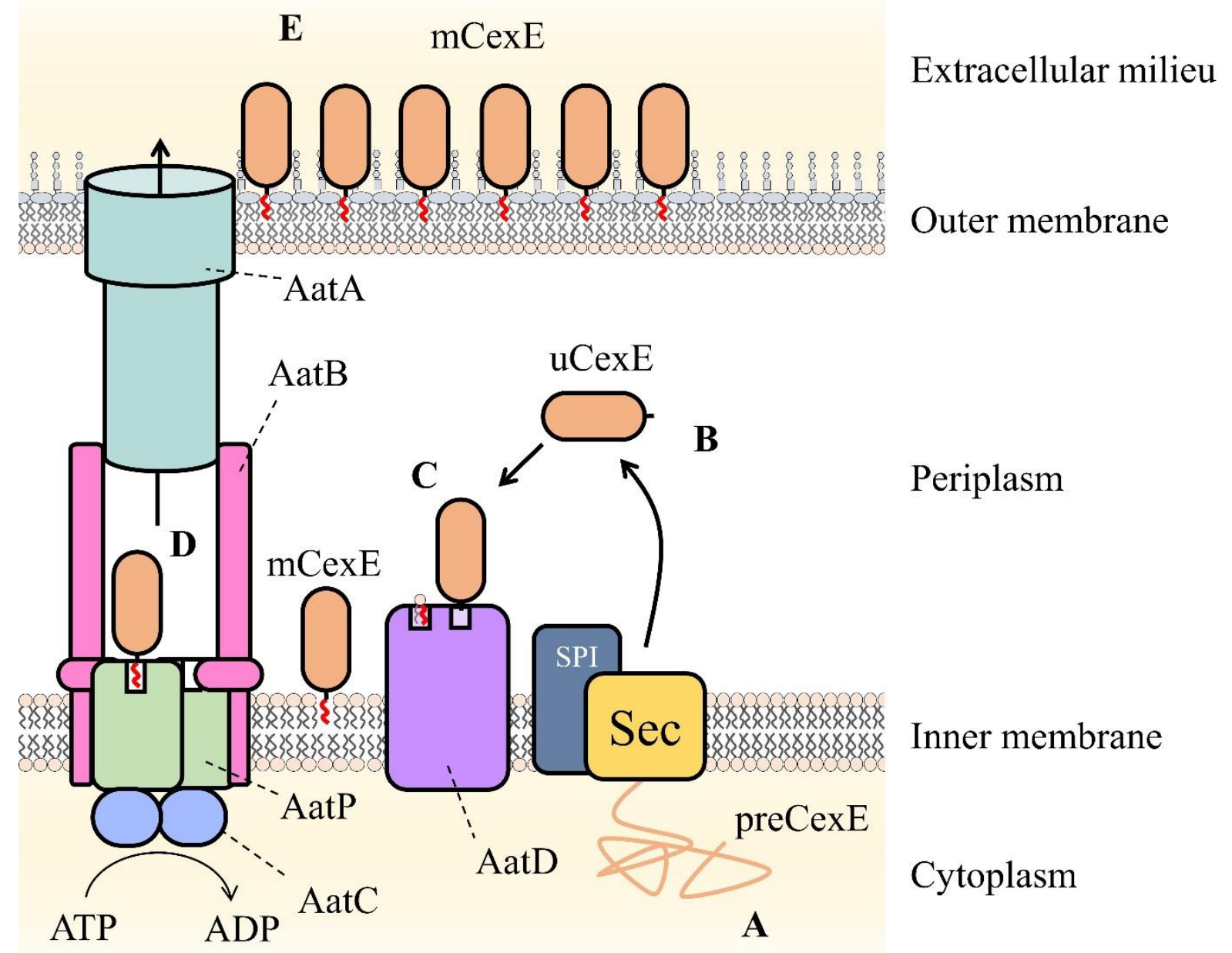

Fig 10. Schematic of proposed Aat system mechanism. A, preCexE produced in the cytoplasm is secreted into the periplasm by the Sec pathway $(\mathrm{Sec})$. B, The signal sequence is cut by signal peptidase I (SPI) resulting in soluble uCexE. C, An acyl chain is added to the N-terminal glycine of uCexE by AatD. Accordingly, mCexE associates with the membrane. D, mCexE is extracted out of the inner membrane by the AatP and AatC complex. A single channel is formed comprised of AatP, AatA, AatB, and AatC that allows the secretion of mCexE. E, mCexE is inserted into the outer leaflet of the outer membrane. mCexE remains associated with the outer membrane by the single acyl chain incorporated onto the N-terminal glycine. 


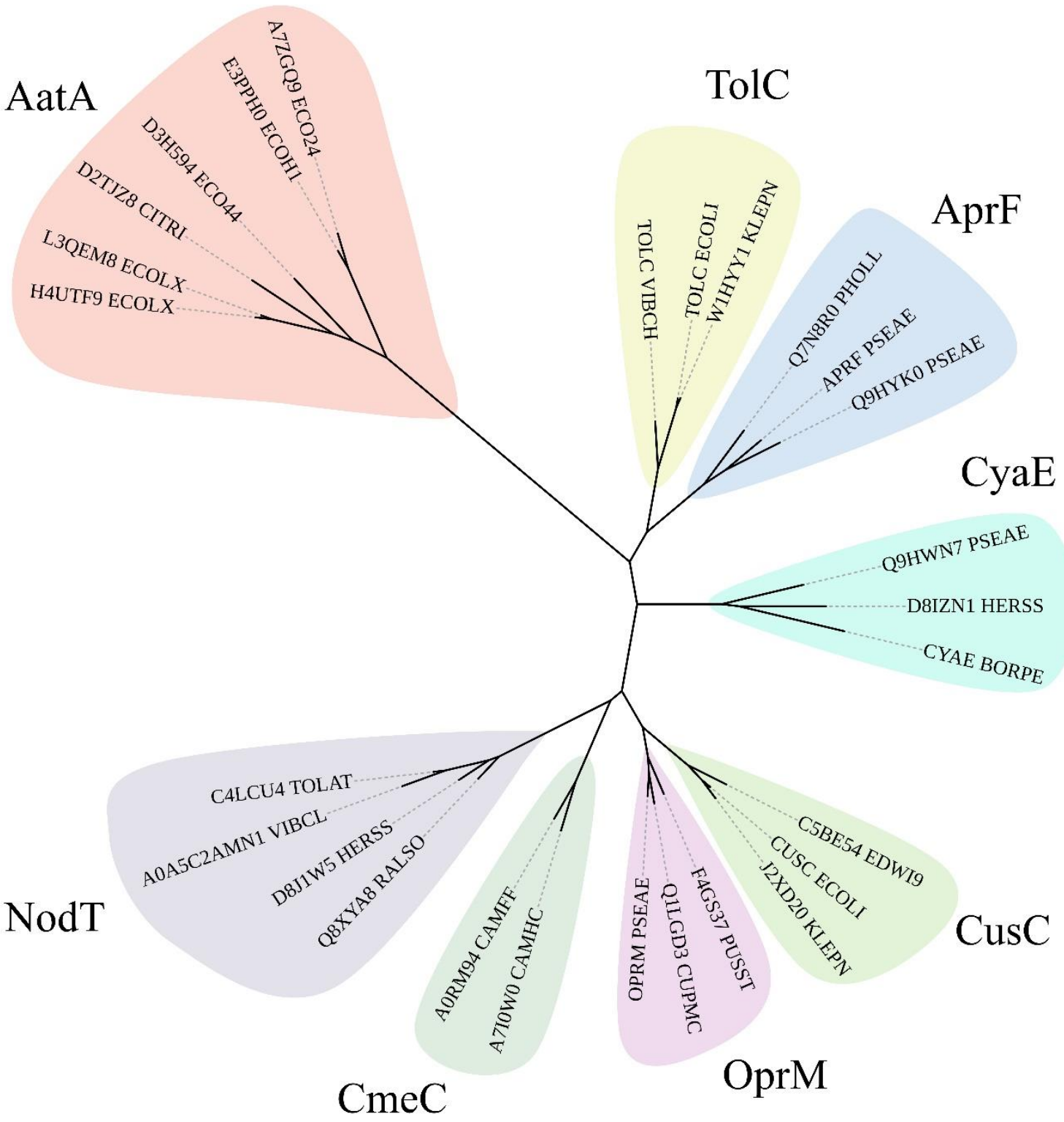

592 Supplementary Fig S1. Phylogram of AatA, TolC and other OMP associated with T1SS and efflux

593 pumps. The HMM of AatA proteins was used to confirm AatA as a homolog of TolC and OMP

594 proteins. Representative sequences of secretion associated OMPs (AprF, CyaE and TolC) and efflux

595 associated OMPs (CusC, OprM, CmeC, and NodT) were used to construct a phylogram. Although AatA

596 is more closely related to the secretion associated OMPs AprF and CyaE than the efflux associated

597 OMPs. TolC is a promiscuous protein that functions as the OMP for secretion and efflux systems. All

598 Phylograms were constructed using RAxML and drawn using iTOL. 


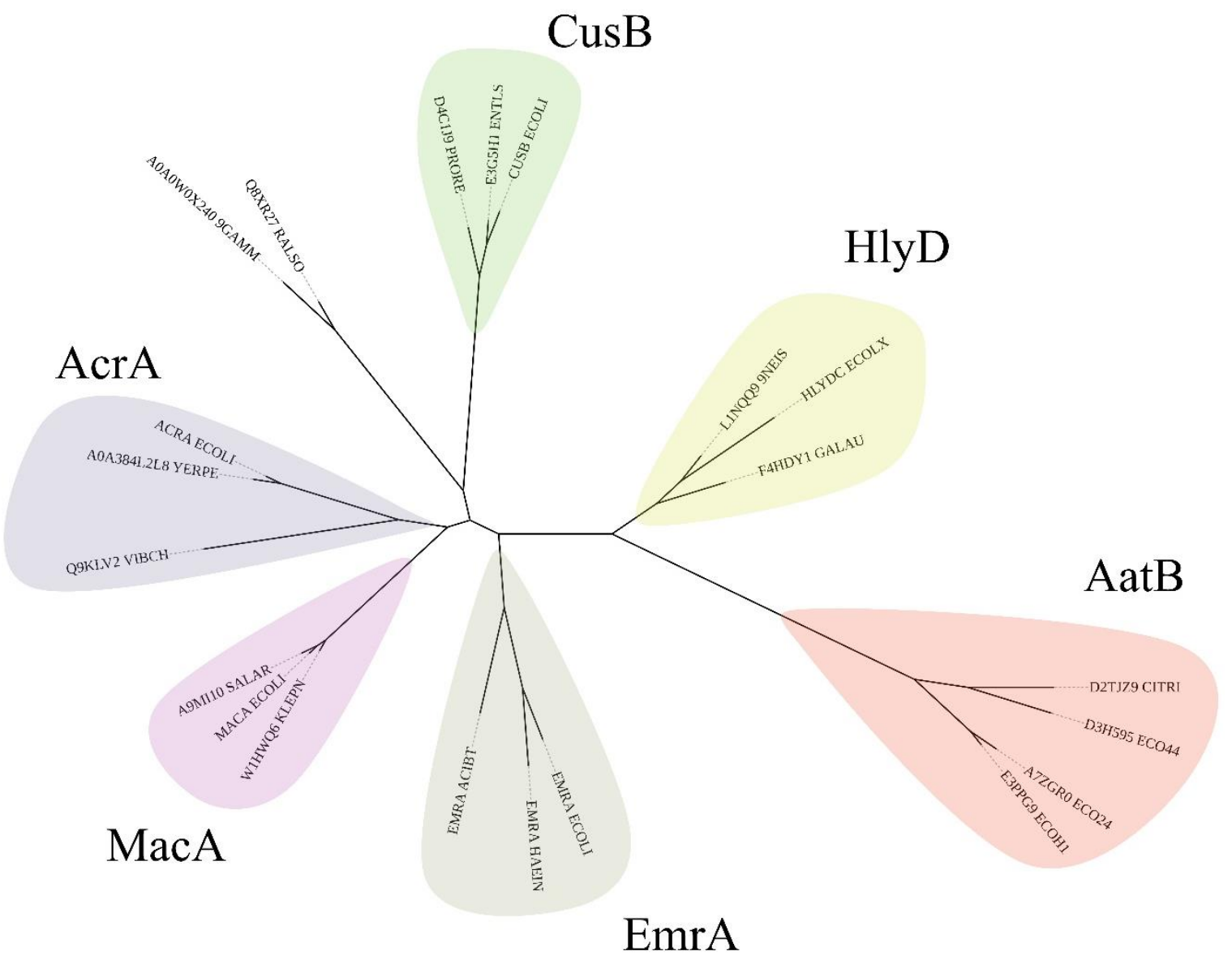

Supplementary Fig S2. Phylogram of AatB and PAPs involved in T1SS and efflux pumps. The

601 AatB HMM identified it as a homolog of the periplasmic adaptor proteins. Representative sequences of

602 PAPs involved in T1SS and efflux were used to construct a phylogenetic tree using RAxML. The

603 resulting tree was drawn in iTOL. AatB clades with the T1SS PAP HlyD which is involved in haemolysin secretion instead of the efflux associated PAPs of EmrA, MacA, AcrA, and CusB. 


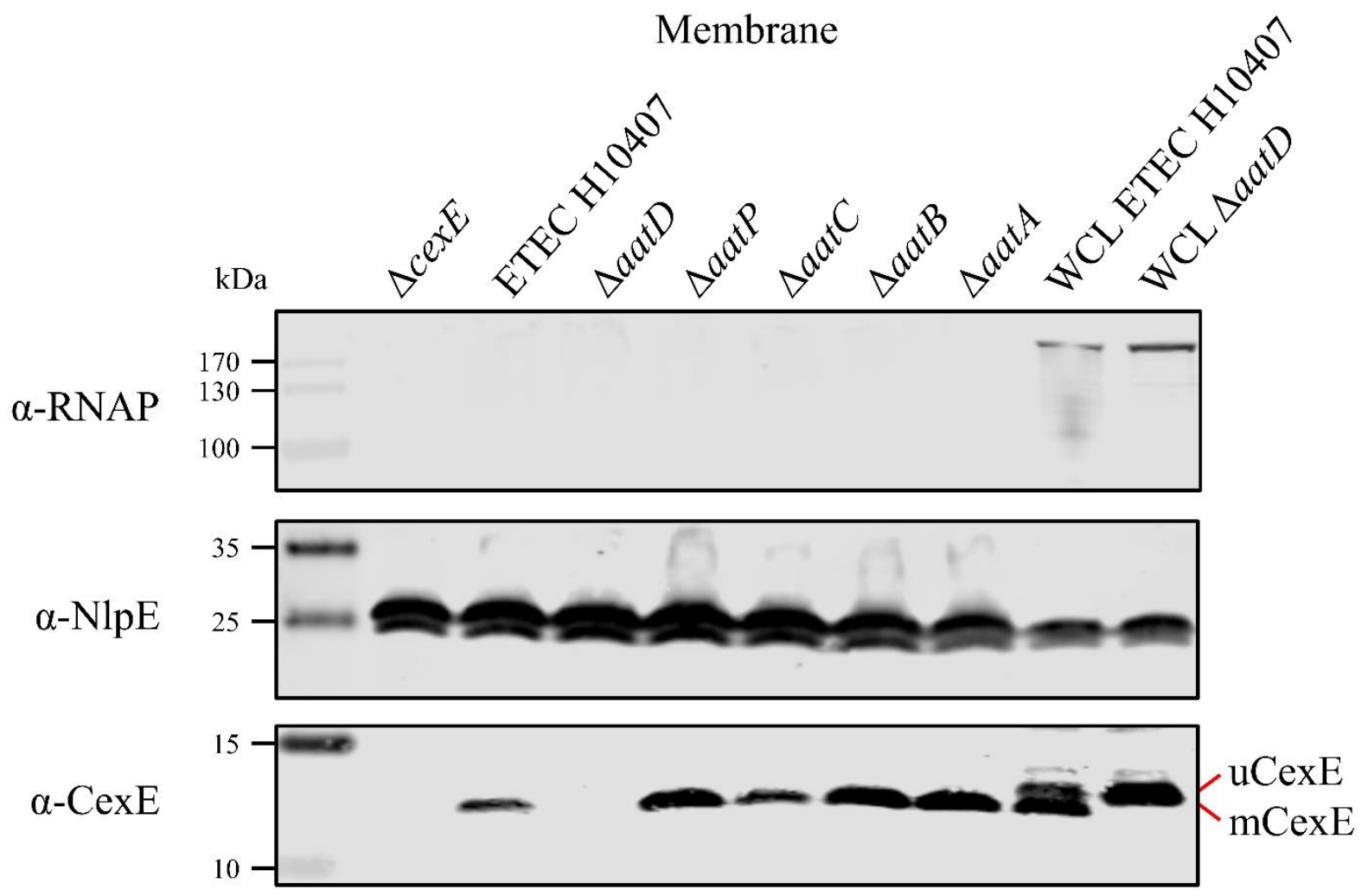

607 Supplementary Fig S3. Membrane localisation of CexE. The membrane fraction of ETEC H10407

608 and aat mutant harbouring $\mathrm{pCfaD}$ were separated by SDS-PAGE. Transferred to nitrocellulose and 

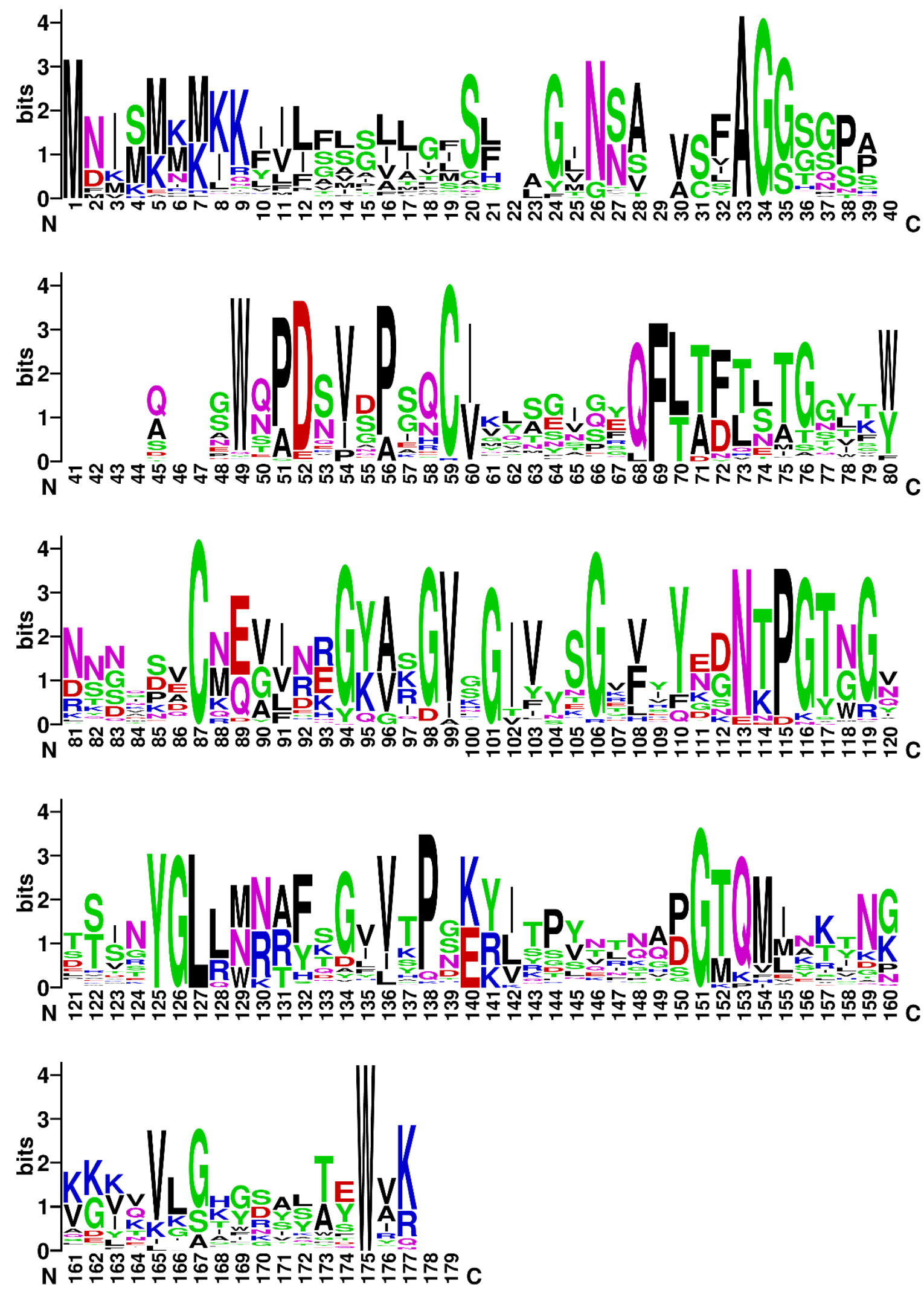

Supplementary Fig S4. WebLogo of Aap and CexE sequences. Aap and CexE sequences were aligned using ClustalO. The alignment was then placed into WebLogo. 
Supplementary Table S3. Bacterial strains used in this study

\begin{tabular}{|c|c|c|}
\hline Strain & Description & Source \\
\hline ETEC H10407 & $\begin{array}{l}\text { Prototypical enterotoxigenic strain. } \\
\mathrm{CFA} / 1^{+}, \mathrm{LT}^{+}, \mathrm{ST}^{+}\end{array}$ & Evans and Evans (1973) \\
\hline ETEC H10407هaatP & aatP deletion mutant & This study \\
\hline ETEC H10407هaatA & aatA deletion mutant & This study \\
\hline ETEC H10407هaatB & aatB deletion mutant & This study \\
\hline ETEC H10407هaatC & aatC deletion mutant & This study \\
\hline ETEC H10407هaatD & aatD deletion mutant & This study \\
\hline ETEC H10407هcexE & cexE deletion mutant & This study \\
\hline E. coli $\mathrm{DH} 5 \alpha$ & Cloning strain & NEB \\
\hline E. coli BL21(DE3) & Protein production strain & Invitrogen \\
\hline EAEC 042 & $\begin{array}{l}\text { Prototypical enteroaggregative strain. } \\
\text { AAF } 1+\text {, EAST } 1+\text {, Pet+ }\end{array}$ & Nataro et al. (1985) \\
\hline EAEC 042 $\Delta a a p$ & $\begin{array}{l}\text { EAEC } 042 \text { with pJB5603 inserted into } \\
\text { aap }\end{array}$ & Sheikh et al. (2002) \\
\hline EAEC 042 $\triangle a a t D$ & $\begin{array}{l}\text { EAEC } 042 \text { with pJB5603 inserted into } \\
\text { aatD }\end{array}$ & Nishi et al. (2003) \\
\hline
\end{tabular}


Supplementary Table S4: Primers used in this study.

\begin{tabular}{|c|c|c|}
\hline Name & Sequence & Description \\
\hline CcexE-F & $\begin{array}{l}\text { GGCGGCCATATGAAAAAATATATATTAG } \\
\text { GTGT }\end{array}$ & Cloning cexE from ETEC H10407 into pET26(b) \\
\hline CcexE-R & $\begin{array}{l}\text { CGAAGCCTCGAGTTTATACCAATAAGGG } \\
\text { GTGT }\end{array}$ & Cloning cexE from ETEC H10407 into pET26(b) \\
\hline GDaatP-F & $\begin{array}{l}\text { GAATTCAAGCTTGCAAAGCGTATTGTTG } \\
\text { GTGCAGGCTTGTATAAGTTATATGGACC } \\
\text { GGTCAATTGGCTGGAG }\end{array}$ & $\begin{array}{l}\text { Forward primer for generating kanamycin cassette and } \\
\text { disruption of aatP gene in ETEC H10407 }\end{array}$ \\
\hline GDaatP-R & $\begin{array}{l}\text { GAGCTCCATATGAATATAAATATAATCT } \\
\text { TCATGAAAATCTTTCTTTTATTAAAATA } \\
\text { TCCTCCTTAGTTCC }\end{array}$ & $\begin{array}{l}\text { Reverse primer for generating kanamycin cassette and } \\
\text { disruption of aatP gene in ETEC H10407 }\end{array}$ \\
\hline GDaatA-F & $\begin{array}{l}\text { GAATTCAAGCTTGATTTATCAATCTTAA } \\
\text { TAAAAGAAAGATTTCATGAAGAGACCG } \\
\text { GTCAATTGGCTGGAG }\end{array}$ & $\begin{array}{l}\text { Forward primer for generating kanamycin cassette and } \\
\text { disruption of aatA gene in ETEC H10407 }\end{array}$ \\
\hline GDaatA-R & $\begin{array}{l}\text { GAGCTCCATATGTATATAATTCAATTTC } \\
\text { ATTTTCCTTTTTTATTAACTCTCAATAT } \\
\text { CCTCCTTAGTTCC }\end{array}$ & $\begin{array}{l}\text { Reverse primer for generating kanamycin cassette and } \\
\text { disruption of aatA gene in ETEC H10407 }\end{array}$ \\
\hline GDaatB-F & $\begin{array}{l}\text { GAATTCAAGCTTGAATTATATAAAATGC } \\
\text { ATATGTTTTTTATTGGTTAGTTTGACCG } \\
\text { GTCAATTGGCTGGAG }\end{array}$ & $\begin{array}{l}\text { Forward primer for generating kanamycin cassette and } \\
\text { disruption of aatB gene in ETEC H10407 }\end{array}$ \\
\hline GDaatB-R & $\begin{array}{l}\text { GAGCTCCATATGATCAATACTTAATTTA } \\
\text { ATCATGTAGTGTTATCTCAAATGCTGAA } \\
\text { TATCCTCCTTAGTTCC }\end{array}$ & $\begin{array}{l}\text { Reverse primer for generating kanamycin cassette and } \\
\text { disruption of } a a t B \text { gene in ETEC H10407 }\end{array}$ \\
\hline GDaatC-F & $\begin{array}{l}\text { GAATTCAAGCTTCTTGGATACAGCATTT } \\
\text { GAGATAACACTACATGATTAAATTAGAC } \\
\text { CGGTCAATTGGCTGGAG }\end{array}$ & $\begin{array}{l}\text { Forward primer for generating kanamycin cassette and } \\
\text { disruption of aatC gene in ETEC } \mathrm{H} 10407\end{array}$ \\
\hline GDaatC-R & $\begin{array}{l}\text { GAGCTCCATATGAAATCACATAAAATTT } \\
\text { TTATTAAAAGGATATAACCTTCATAATA } \\
\text { TCCTCCTTAGTTCC }\end{array}$ & $\begin{array}{l}\text { Reverse primer for generating kanamycin cassette and } \\
\text { disruption of } a a t C \text { gene in ETEC } \mathrm{H} 10407\end{array}$ \\
\hline GDaatD-F & $\begin{array}{l}\text { GAATTCAAGCTTTCGTATATTGGCATTA } \\
\text { AATTATCTTTTGAATTGATTAATGGACC } \\
\text { GGTCAATTGGCTGGAG }\end{array}$ & $\begin{array}{l}\text { Forward primer for generating kanamycin cassette and } \\
\text { disruption of aatD gene in ETEC H10407 }\end{array}$ \\
\hline GDaatD-R & $\begin{array}{l}\text { GAGCTCCATATGACCAAATGATGTATAT } \\
\text { GGTTTATACATCAATGACAAAAAAAATA } \\
\text { TCCTCCTTAGTTCC }\end{array}$ & $\begin{array}{l}\text { Reverse primer for generating kanamycin cassette and } \\
\text { disruption of aatD gene in ETEC H10407 }\end{array}$ \\
\hline cexE-F1 & GGGTTAAGTGATAACAGGCG & Forward flanking primer of cexE gene in ETEC H10407 \\
\hline cexE-R1 & GCACCAACAATACGCTTTGC & Reverse flanking primer of $c e x E$ gene in ETEC H10407 \\
\hline aatP-F1 & TACAATGTCGGGACTCAACC & Forward flanking primer of aatP gene in ETEC H10407 \\
\hline aatP-R1 & ACATACGCAAATGCGGATGG & Reverse flanking primer of aatP gene in ETEC H10407 \\
\hline aatA-F1 & CATCAGGTTGAGTTACTCGC & Forward flanking primer of aatA gene in ETEC H10407 \\
\hline aatA-R1 & CATTGTAAGCATTGCTGGCG & Reverse flanking primer of aatA gene in ETEC H10407 \\
\hline aatB-F1 & CATTCCGCTAAGTGGATTGC & Forward flanking primer of $a a t B$ gene in ETEC H10407 \\
\hline aatB-R1 & ATATCCGACACATCACTCCC & Reverse flanking primer of $a a t B$ gene in ETEC H10407 \\
\hline aatC-F1 & GTAATCCACATGTGCAAGGG & Forward flanking primer of aatC gene in ETEC H10407 \\
\hline aatC-R1 & TGCCGTAGTTATTCGTGAGG & Reverse flanking primer of aatC gene in ETEC H10407 \\
\hline aatD-F1 & СTCCCTCTATAGTGTGTAGC & Forward flanking primer of aatD gene in ETEC H10407 \\
\hline aatD-R1 & TCTGGCGTCCATTCATTTCC & Reverse flanking primer of aatD gene in ETEC H10407 \\
\hline $\mathrm{RM} \_c e x E \_3-\mathrm{RV}$ & ACCACCACCCGCAATCG & $\begin{array}{l}\text { Remove all of cexE apart from the last } 3 \mathrm{~N} \text {-terminal } \\
\text { glycine residues }\end{array}$ \\
\hline $\mathrm{RM} \_c e x E \_2-\mathrm{RV}$ & ACCACCCGCAATCGCGC & $\begin{array}{l}\text { Remove all of cexE apart from the last } 2 \mathrm{~N} \text {-terminal } \\
\text { glycine residues }\end{array}$ \\
\hline RM_cexE_1-RV & ACCCGCAATCGCGCTCAGG & $\begin{array}{l}\text { Remove all of } c \operatorname{ex} E \text { apart from the last } 1 \mathrm{~N} \text {-terminal } \\
\text { glycine residues }\end{array}$ \\
\hline
\end{tabular}


bioRxiv preprint doi: https://doi.org/10.1101/2020.09.23.310854; this version posted October 5, 2020. The copyright holder for this preprint (which was not certified by peer review) is the author/funder, who has granted bioRxiv a license to display the preprint in perpetuity. It is made available under aCC-BY-NC-ND 4.0 International license.

\begin{tabular}{|c|c|c|}
\hline $\mathrm{RM} \_c e x E-\mathrm{RV}$ & CGCAATCGCGCTCAGGCTACC & Remove all of cexE apart from the signal sequence. \\
\hline RM_cexE-FW & GTTAGCAAGGGCGAGGAAGATAAC & $\begin{array}{l}\text { Forward primer for removing cexE from CexE-mCherry } \\
\text { fusion. }\end{array}$ \\
\hline G20A-F & TCCGCGATAGCTGCAGGCGGTAATTC & Substitution mutant G20A of $\operatorname{cexE}$ \\
\hline G20A-R & GAATTACCGCCTGCAGCTATCGCGGA & Substitution mutant G20A of $c e x E$ \\
\hline G20E-F & TCCGCGATAGCTGAAGGCGGTAATTC & Substitution mutant G20E of cexE \\
\hline G20E-R & GAATTACCGCCTTCAGCTATCGCGGA & Substitution mutant G20E of cexE \\
\hline G21A-F & $\begin{array}{l}\text { GCGATAGCTGGAGCCGGTAATTCTGAAC } \\
\text { G }\end{array}$ & Substitution mutant G21A of $c e x E$ \\
\hline G21A-R & $\begin{array}{l}\text { CGTTCAGAATTACCGGCTCCAGCTATCG } \\
\text { C }\end{array}$ & Substitution mutant G21A of $c e x E$ \\
\hline G21E-F & $\begin{array}{l}\text { GCGATAGCTGGAGAAGGTAATTCTGAAC } \\
\text { G }\end{array}$ & Substitution mutant G21E of cexE \\
\hline G21E_R & $\begin{array}{l}\text { CGTTCAGAATTACCTTCTCCAGCTATCG } \\
\text { C }\end{array}$ & Substitution mutant G21E of cexE \\
\hline G22A-F & GATAGCTGGAGGCGCTAATTCTGAACG & Substitution mutant G22A of cexE \\
\hline G22A-R & CGTTCAGAATTAGCGCCTCCAGCTATC & Substitution mutant G22A of $c e x E$ \\
\hline G22E-F & GATAGCTGGAGGCGAGAATTCTGAACG & Substitution mutant G22E of $c e x E$ \\
\hline G22E-R & CGTTCAGAATTCTCGCCTCCAGCTATC & Substitution mutant G22E of $c e x E$ \\
\hline N23A-RH-F & GCGTCTGAACGACCGCCTTCCG & Substitution mutant N23A of $c e x E$ \\
\hline N23E-RH-F & GAATCTGAACGACCGCCTTCCG & Substitution mutant N23E of $c e x E$ \\
\hline N23-RH-R & ACCGCCTCCAGCTATCGCG & $\begin{array}{l}\text { Reverse primer for both } \mathrm{N} 23 \mathrm{~A} \text { and } \mathrm{N} 23 \mathrm{E} \text { substitution } \\
\text { mutants }\end{array}$ \\
\hline S24A-F & GGAGGCGGTAATGCTGAACGACCGCC & Substitution mutant S24A of cexE \\
\hline S24A-R & GGAGGCGGTAATGCTGAACGACCGCC & Substitution mutant S24A of cexE \\
\hline S24E-F & GGAGGCGGTAATGAAGAACGACCGCC & Substitution mutant S24E of $c e x E$ \\
\hline S24E-R & GGCGGTCGTTCTTCATTACCGCCTCC & Substitution mutant S24E of cexE \\
\hline H10407_E217A-FW & $\begin{array}{l}\text { CGACATCGATATGGTGGTTTTTAGCGCG } \\
\text { AACACCGTGTACGGTTTCAAGAACCAG }\end{array}$ & Substitution mutant E217A of aatD \\
\hline H10407_E217A-RV & $\begin{array}{l}\text { CTGGTTCTTGAAACCGTACACGGTGTTC } \\
\text { GCGCTAAAAACCACCATATCGATGTCG }\end{array}$ & Substitution mutant E217A of $a a t D$ \\
\hline H10407_K278A-FW & $\begin{array}{l}\text { CTATTACAAAGACAACTTCCTGATCAAC } \\
\text { CAAGCGAAAGCGCTGATTCCGTTTGTTG } \\
\text { AGCAGC }\end{array}$ & Substitution mutant K278A of aatD \\
\hline H10407_K278A-RV & $\begin{array}{l}\text { GCTGCTCAACAAACGGAATCAGCGCTTT } \\
\text { CGCTTGGTTGATCAGGAAGTTGTCTTTG } \\
\text { TAATAG }\end{array}$ & Substitution mutant K278A of aatD \\
\hline H10407_E286A-FW & $\begin{array}{l}\text { GCGCTGATTCCGTTTGTTGCGCAGCAAT } \\
\text { GGTTCTTTAGC }\end{array}$ & Substitution mutant E286A of $a a t D$ \\
\hline H10407_E286A-RV & $\begin{array}{l}\text { GCTAAAGAACCATTGCTGCGCAACAAAC } \\
\text { GGAATCAGCGC }\end{array}$ & Substitution mutant E286A of $a a t D$ \\
\hline H10407_C325A-FW & $\begin{array}{l}\text { CAACCACAACGGTATCAACATTAAGACC } \\
\text { TATATTGCGTACGATGTTCTGTTCCCGG } \\
\text { AAACCCATAAAAGC }\end{array}$ & Substitution mutant C325A of aatD \\
\hline H10407_C325A-RV & $\begin{array}{l}\text { GCTTTTATGGGTTTCCGGGAACAGAACA } \\
\text { TCGTACGCAATATAGGTCTTAATGTTGA } \\
\text { TACCGTTGTGGTTG }\end{array}$ & Substitution mutant C325A of aatD \\
\hline $\mathrm{T} 7 \mathrm{~F}$ & TAATACGACTCACTATAGGG & $\begin{array}{l}\text { Forward primer for amplification of pET } 26 \mathrm{~b}(+) \text { multiple } \\
\text { cloning site }\end{array}$ \\
\hline T7R & GCTAGTTATTGCTCAGCGG & $\begin{array}{l}\text { Reverse primer for amplification of pET26b }(+) \text { multiple } \\
\text { cloning site }\end{array}$ \\
\hline
\end{tabular}


Supplementary Table S5. Plasmids used in this study

\begin{tabular}{|c|c|c|}
\hline Plasmid & Description & Source \\
\hline pDOC-K & contains bla and kanR with FRT sites & Lee et al. (2009) \\
\hline pKD46 & $\begin{array}{l}\text { Heat sensitive plasmid with } \lambda \text { Red recombinase genes } \\
\text { expressed in response to L-arabinose }\end{array}$ & $\begin{array}{l}\text { Datsenko and } \\
\text { Wanner (2000) }\end{array}$ \\
\hline pCP20 & Heat sensitive plasmid encoding Flp recombinase gene & $\begin{array}{l}\text { Datsenko and } \\
\text { Wanner (2000) }\end{array}$ \\
\hline pET26b $(+)$ & $\begin{array}{l}\text { T7 expression vector with C-terminal 6His tag, IPTG } \\
\text { inducible, } k a n R\end{array}$ & Novagen \\
\hline pET26b-cexE & pET26b with cexE from ETEC H10407 & This study \\
\hline pBAD/myc-HisA & $\begin{array}{l}\text { L-arabinose expression vector with C-terminal Myc } \\
\text { and } 6 \mathrm{His} \text { tag }\end{array}$ & Invitrogen \\
\hline pJNW & $\begin{array}{l}\text { Complete aat gene cluster from EAEC } 042 \text { cloned into } \\
\text { pZC } 320\end{array}$ & $\begin{array}{l}\text { Nishi et al. } \\
\text { (2003) }\end{array}$ \\
\hline $\mathrm{pCfaD}$ & $\begin{array}{l}\text { pBAD/myc-HisA with } c f a D \text { under PBAD from ETEC } \\
\text { H10407 }\end{array}$ & This study \\
\hline pACYC184 & Plasmid with p15A origin of replication & $\begin{array}{l}\text { Chang and } \\
\text { Cohen (1978) }\end{array}$ \\
\hline pACYC-cexE-6His & $\begin{array}{l}\text { pACYC } 184 \text { with } c e x E \text { under the control of the native } \\
\text { cexE promoter with a C-terminal } 6 \text { His tag }\end{array}$ & This study \\
\hline pACYCDuet-1 & $\begin{array}{l}\text { Plasmid with p15A origin of replication and two } \mathrm{T} 7 \\
\text { promoters. }\end{array}$ & Novagen \\
\hline pACYC-aatD & $\begin{array}{l}\text { The ETEC H10407 aatD gene was synthesised by } \\
\text { Genscript and transformed in pACYCDuet- } 1\end{array}$ & This study \\
\hline pRSF-GGG-mCherry & $\begin{array}{l}\text { mCherry with three glycine residues at the } \mathrm{N} \text {-terminus } \\
\text { after the CexE signal sequence }\end{array}$ & This study \\
\hline pRSF-GG-mCherry & $\begin{array}{l}\text { mCherry with two glycine residues at the } \mathrm{N} \text {-terminus } \\
\text { after the CexE signal sequence }\end{array}$ & This study \\
\hline pRSF-G-mCherry & $\begin{array}{l}\text { mCherry with one glycine residue at the } \mathrm{N} \text {-terminus } \\
\text { after the CexE signal sequence }\end{array}$ & This study \\
\hline pRSF-SS-mCherry & mCherry with the CexE signal sequence & This study \\
\hline
\end{tabular}


Altschul, S. F., Madden, T. L., Schäffer, A. A., Zhang, J., Zhang, Z., Miller, W., \& Lipman, D. J. (1997). Gapped BLAST and PSI-BLAST : a new generation of protein database search programs. Nucleic Acids Res, 25(17), 3389-3402.

Babu, M. M., Priya, M. L., Selvan, A. T., Madera, M., Gough, J., Aravind, L., \& Sankaran, K. (2006). A database of bacterial lipoproteins (DOLOP) with functional assignments to predicted lipoproteins. J Bacteriol, 188(8), 2761-2773. https://doi.org/10.1128/JB.188.8.2761-2773.2006

Baldi, D. L., Higginson, E. E., Hocking, D. M., Praszkier, J., Cavaliere, R., James, C. E., BennettWood, V., Azzopardi, K. I., Turnbull, L., Lithgow, T., Robins-Browne, R. M., Whitchurch, C. B., \& Tauschek, M. (2012). The type II secretion system and its ubiquitous lipoprotein substrate, $\mathrm{SslE}$, are required for biofilm formation and virulence of enteropathogenic Escherichia coli. Infect Immun, 80(6), 2042-2052. https://doi.org/10.1128/IAI.06160-11

Baudry, B., Savarino, S. J., Vial, P., Kaper, J. B., \& Levine, M. M. (1990). A Sensitive and Specific DNA Probe to Identify Enteroaggregative Escherichia coli, a Recently Discovered Diarrheal Pathogen. J Infect Dis, 161(6), 1249-1251.

Belmont-Monroy, L., Saitz-rojas, W., Soria-bustos, J., Mickey, S., Sherman, N. E., Orsburn, B. C., Ruiz-perez, F., \& Santiago, A. E. (2020). Characterization of a novel AraC/XylS-regulated family of N-acyltransferases in pathogens of the order Enterobacterales. PLoS Pathog, 16(8), e1008776. https://doi.org/10.1371/journal.ppat.1008776

Chang, A. C. Y., \& Cohen, S. N. (1978). Construction and characterization of amplifiable multicopy DNA cloning vehicles derived from the P15A cryptic miniplasmid. J Bacteriol, 134(3), 11411156. https://doi.org/10.1128/jb.134.3.1141-1156.1978

Cowles, C. E., Li, Y., Semmelhack, M. F., Cristea, I. M., \& Silhavy, T. J. (2012). The free and bound forms of Lpp occupy distinct subcellular locations in Escherichia coli. Mol Microbiol, 79(5), 1168-1181. https://doi.org/10.1111/j.1365-2958.2011.07539.x.The

Crossman, L. C., Chaudhuri, R. R., Beatson, S. A., Wells, T. J., Desvaux, M., Cunningham, A. F., Petty, N. K., Mahon, V., Brinkley, C., Hobman, J. L., Savarino, S. J., Turner, S. M., Pallen, M. J., Penn, C. W., Parkhill, J., Turner, A. K., Johnson, T. J., Thomson, N. R., Smith, S. G. J., \& Henderson, I. R. (2010). A commensal gone bad: complete genome sequence of the prototypical enterotoxigenic Escherichia coli strain H10407. J Bacteriol, 192(21), 5822-5831. https://doi.org/10.1128/JB.00710-10

Crow, A., Greene, N. P., Kaplan, E., \& Koronakis, V. (2017). Structure and mechanotransmission mechanism of the MacB ABC transporter superfamily. Proc Natl Acad Sci USA, 114(47), 12572-12577. https://doi.org/10.1073/pnas.1712153114

Datsenko, K. a, \& Wanner, B. L. (2000). One-step inactivation of chromosomal genes in Escherichia coli K-12 using PCR products. Proc Natl Acad Sci USA, 97(12), 6640-6645. https://doi.org/10.1073/pnas.120163297

Dev, I. K., Harvey, R. J., \& Ray, P. H. (1985). Inhibition of prolipoprotein signal peptidase by globomycin. J Biol Chem, 260(10), 5891-5894.

Duronio, R. J., Jackson-Machelski, E., Heuckeroth, R. O., Olins, P. O., Devine, C. S., Yonemoto, W., Slice, L. W., Taylor, S. S., \& Gordon, J. I. (1990). Protein N-myristoylation in Escherichia coli: Reconstitution of a eukaryotic protein modification in bacteria. Proc Natl Acad Sci USA, 87(4), 1506-1510. https://doi.org/10.1073/pnas.87.4.1506

Evans, D. J., \& Evans, D. G. (1973). Three Characteristics Associated with Enterotoxigenic Escherichia coli Isolated from Man. Infect Immun, 8(3), 322-328.

Finn, R. D., Clements, J., \& Eddy, S. R. (2011). HMMER web server: interactive sequence similarity searching. Nucleic Acids Res, 39(W29), W29-37. https://doi.org/10.1093/nar/gkr367

Fitzpatrick, A. W. P., Llabrés, S., Neuberger, A., Blaza, J. N., Bai, X., Okada, U., Murakami, S., Veen, H. W. Van, Zachariae, U., Scheres, S. H. W., Luisi, B. F., \& Du, D. (2017). Structure of the MacAB-TolC ABC-type tripartite multidrug efflux pump. Nat Microbiol, 2, 17070. https://doi.org/10.1038/nmicrobiol.2017.70

Gélis-Jeanvoine, S., Lory, S., Oberto, J., \& Buddelmeijer, N. (2015). Residues located on membrane- 
embedded flexible loops are essential for the second step of the apolipoprotein $\mathrm{N}$-acyltransferase reaction. Mol Microbiol, 95(4), 692-705. https://doi.org/10.1111/mmi.12897

Grabowicz, M. (2019). Lipoproteins and Their Trafficking to the Outer Membrane. EcoSal Plus, 8(2), 1-8. https://doi.org/10.1128/ecosalplus.esp-0038-2018

Gupta, S. D., Gan, K., Schmid, M. B., \& Wu, H. C. (1993). Characterization of a temperaturesensitive mutant of Salmonella typhimurium defective in apolipoprotein $\mathrm{N}$-acyltransferase. $J$ Biol Chem, 268(22), 16551-16556.

Hancock, J. F., Magee, A. I., Childs, J. E., \& Marshall, C. J. (1989). All ras proteins are polyisoprenylated but only some are palmitoylated. Cell, 57(7), 1167-1177. https://doi.org/10.1016/0092-8674(89)90054-8

Hodson, C., Yang, J., Hocking, D. M., Azzopardi, K., Chen, Q., Holien, J. K., Parker, M. W., Tauschek, M., \& Robins-browne, R. M. (2017). Control of Virulence Gene Expression by the Master Regulator, CfaD, in the Prototypical Enterotoxigenic Escherichia coli Strain, H10407. Front Microbiol, 11(8), 1525. https://doi.org/10.3389/fmicb.2017.01525

Hooda, Y., Lai, C. C. L., Judd, A., Buckwalter, C. M., Shin, H. E., Gray-Owen, S. D., \& Moraes, T. F. (2016). Slam is an outer membrane protein that is required for the surface display of lipidated virulence factors in Neisseria. Nat Microbiol, 1(4), 16009. https://doi.org/10.1038/nmicrobiol.2016.9

Huie, J. L., \& Silhavy, T. J. (1995). Suppression of signal sequence defects and azide resistance in Escherichia coli commonly result from the same mutations in secA. J Bacteriol, 177(12), 35183526.

Kelley, L. A., Mezulis, S., Yates, C. M., Wass, M. N., \& Sternberg, M. J. (2016). The Phyre2 web protal for protein modeling, prediction and analysis. Nat Protoc, 10(6), 845-858. https://doi.org/10.1038/nprot.2015-053

Konovalova, A., Perlman, D. H., Cowles, C. E., \& Silhavy, T. J. (2014). Transmembrane domain of surface-exposed outer membrane lipoprotein RcsF is threaded through the lumen of $\beta$-barrel proteins. Proc Natl Acad Sci USA, 111(41), E4350-E4358. https://doi.org/10.1073/pnas.1417138111

Kurtzhals, P. (2007). Pharmacology of Insulin Detemir. Endocrinol Metab Clin North Am, 36(1), 14 20. https://doi.org/10.1016/S0889-8529(07)80004-1

Lee, D. J., Bingle, L. E. H., Heurlier, K., Pallen, M. J., Penn, C. W., Busby, S. J. W., \& Hobman, J. L. (2009). Gene doctoring: a method for recombineering in laboratory and pathogenic Escherichia coli strains. BMC Microbiol, 9, 252. https://doi.org/10.1186/1471-2180-9-252

Letunic, I., \& Bork, P. (2019). Interactive Tree of Life (iTOL) v4: Recent updates and new developments. Nucleic Acids Res, 47(W1), 256-259. https://doi.org/10.1093/nar/gkz239

Madeira, F., Park, Y. M., Lee, J., Buso, N., Gur, T., Madhusoodanan, N., Basutkar, P., Tivey, A. R. N., Potter, S. C., Finn, R. D., \& Lopez, R. (2019). The EMBL-EBI search and sequence analysis tools APIs in 2019. Nucleic Acids Res, 47(W1), W636-W641. https://doi.org/10.1093/nar/gkz268

Martin, D. D. O., Beauchamp, E., \& Berthiaume, L. G. (2011). Post-translational myristoylation: Fat matters in cellular life and death. Biochimie, 93(1), 18-31. https://doi.org/10.1016/j.biochi.2010.10.018

Masao Tokunaga, Hiroko Tokunaga, \& Wu, H. C. (1984). Post-translational modification and processing of outer membrane prolipoproteins in Escherichia coli. Mol Cell Biochem, 60(1), 515. https://doi.org/10.1007/BF00226297

Moore, S. D., \& Prevelige, P. E. (2002). A P22 Scaffold Protein Mutation Increases the Robustness of Head Assembly in the Presence of Excess Portal Protein. J Virol, 76(20), 10245-10255. https://doi.org/10.1128/jvi.76.20.10245-10255.2002

Nakayama, H., Kurokawa, K., \& Lee, B. L. (2012). Lipoproteins in bacteria: structures and biosynthetic pathways. FEBS J, 279(23), 4247-4268. https://doi.org/10.1111/febs.12041

Nataro, J., Baldini, M., Kaper, J., Black, R., Bravo, N., \& Levine, M. (1985). Detection of an adherence factor of enteropathogenic Escherichia coli with a DNA probe. J Infect Dis, 152(3), $560-565$.

Nishi, J., Sheikh, J., Mizuguchi, K., Luisi, B., Burland, V., Boutin, A., Rose, D. J., Blattner, F. R., \& Nataro, J. P. (2003). The export of coat protein from enteroaggregative Escherichia coli by a 
specific ATP-binding cassette transporter system. J Biol Chem, 278(46), 45680-45689. https://doi.org/10.1074/jbc.M306413200

Oliver, D. B., Cabelli, R. J., Dolan, K. M., \& Jarosik, G. P. (1990). Azide-resistant mutants of Escherichia coli alter the SecA protein, an azide-sensitive component of the protein export machinery. Proc Natl Acad Sci USA, 87(21), 8227-8231.

Pepinsky, R. B., Zeng, C., Went, D., Rayhorn, P., Baker, D. P., Williams, K. P., Bixler, S. A., Ambrose, C. M., Garber, E. A., Miatkowski, K., Taylor, F. R., Wang, E. A., \& Galdes, A. (1998). Identification of a palmitic acid-modified form of human Sonic hedgehog. J Biol Chem, 273(22), 14037-14045. https://doi.org/10.1074/jbc.273.22.14037

Petty, N. K., Bulgin, R., Crepin, V. F., Cerdeño-Tárraga, A. M., Schroeder, G. N., Quail, M. a, Lennard, N., Corton, C., Barron, A., Clark, L., Toribio, A. L., Parkhill, J., Dougan, G., Frankel, G., \& Thomson, N. R. (2010). The Citrobacter rodentium genome sequence reveals convergent evolution with human pathogenic Escherichia coli. J Bacteriol, 192(2), 525-538. https://doi.org/10.1128/JB.01144-09

Pilonieta, M. C., Bodero, M. D., \& Munson, G. P. (2007). CfaD-dependent expression of a novel extracytoplasmic protein from enterotoxigenic Escherichia coli. J Bacteriol, 189(14), 5060 5067. https://doi.org/10.1128/JB.00131-07

Resh, M. D. (1994). Myristylation and palmitylation of Src family members: The fats of the matter. Cell, 76(3), 411-413. https://doi.org/10.1016/0092-8674(94)90104-X

Rivas, Z. P., Talbot, K. M., Merselis, L. C., McCormack, R. M., Adkins, B., \& Munson, G. P. (2020). CexE Is a Coat Protein and Virulence Factor of Diarrheagenic Pathogens. Front Microbiol, 11(1374), 1-13. https://doi.org/10.3389/fmicb.2020.01374

Schägger, H. (2006). Tricine-SDS-PAGE. Nat Protoc, 1(1), 16-22. https://doi.org/10.1038/nprot.2006.4

Sheikh, J., Czeczulin, J. R., Harrington, S., Hicks, S., Henderson, I. R., Le Bouguénec, C., Gounon, P., Phillips, A., \& Nataro, J. P. (2002). A novel dispersin protein in enteroaggregative Escherichia coli. J Clin Invest, 110(9), 1329-1337. https://doi.org/10.1172/JCI16172

Stamatakis, A. (2014). RAxML version 8 : a tool for phylogenetic analysis and post-analysis of large phylogenies. Bioinformatics, 30(9), 1312-1313. https://doi.org/10.1093/bioinformatics/btu033

The UniProt Consortium. (2019). UniProt: A worldwide hub of protein knowledge. Nucleic Acids Res, 47, D506-D515. https://doi.org/10.1093/nar/gky1049

Thompson, B. J., Widdick, D. A., Hicks, M. G., Chandra, G., Sutcliffe, I. C., Palmer, T., \& Hutchings, M. I. (2010). Investigating lipoprotein biogenesis and function in the model Grampositive bacterium Streptomyces coelicolor. Mol Microbiol, 77(4), 943-957. https://doi.org/10.1111/j.1365-2958.2010.07261.x

Veronese, F. D., Copeland, T. D., Oroszlan, S., Gallo, R. C., \& Sarngadharan, M. G. (1988). Biochemical and immunological analysis of human immunodeficiency virus gag gene products p17 and p24. J Virol, 62(3), 795-801. https://doi.org/10.1128/jvi.62.3.795-801.1988

Vidal-Ingigliardi, D., Lewenza, S., \& Buddelmeijer, N. (2007). Identification of Essential Residues in Apolipoprotein N-Acyl Transferase, a Member of the CN Hydrolase Family. J Bacteriol, 189(12), 4456-4464. https://doi.org/10.1128/JB.00099-07

Widdick, D. A., Hicks, M. G., Thompson, B. J., Tschumi, A., Chandra, G., Sutcliffe, I. C., Brülle, J. K., Sander, P., Palmer, T., \& Hutchings, M. I. (2011). Dissecting the complete lipoprotein biogenesis pathway in Streptomyces scabies. Mol Microbiol, 80(5), 1395-1412. https://doi.org/10.1111/j.1365-2958.2011.07656.x

Wiktor, M., Weichert, D., Howe, N., Huang, C. Y., Olieric, V., Boland, C., Bailey, J., Vogeley, L., Stansfeld, P. J., Buddelmeijer, N., Wang, M., \& Caffrey, M. (2017). Structural insights into the mechanism of the membrane integral $\mathrm{N}$-acyltransferase step in bacterial lipoprotein synthesis. Nat Commun, 8, 15952. https://doi.org/10.1038/ncomms15952

Yamanaka, H., Kobayashi, H., Takahashi, E., \& Okamoto, K. (2008). MacAB is involved in the secretion of Escherichia coli heat-stable enterotoxin II. J Bacteriol, 190(23), 7693-7698. https://doi.org/10.1128/JB.00853-08

Zückert, W. R. (2014). Secretion of bacterial lipoproteins: through the cytoplasmic membrane, the periplasm and beyond. Biochim Biophys Acta, 1843(8), 1509-1516. https://doi.org/10.1016/j.bbamcr.2014.04.022 
bioRxiv preprint doi: https://doi.org/10.1101/2020.09.23.310854; this version posted October 5, 2020. The copyright holder for this preprint (which was not certified by peer review) is the author/funder, who has granted bioRxiv a license to display the preprint in perpetuity. It is made available under aCC-BY-NC-ND 4.0 International license.

783

784 